\title{
100 years of Bacillus Calmette- Guérin immunotherapy: from cattle to COVID-19
}

\section{Niyati Lobo, Nathan A. Brooks, Alexandre R. Zlotta, Jeffrey D. Cirillo, \\ Stephen Boorjian, Peter C. Black, Joshua J. Meeks, Trinity J. Bivalacqua, Paolo Gontero, Gary D. Steinberg, David McConkey, Marko Babjuk, J. Alfred Witjes and Ashish M. Kamat}

Abstract | Bacillus Calmette-Guérin (BCG) is the most widely used vaccine worldwide and has been used to prevent tuberculosis for a century. BCG also stimulates an anti-tumour immune response, which urologists have harnessed for the treatment of non-muscle-invasive bladder cancer. A growing body of evidence indicates that $\mathrm{BCG}$ offers protection against various non-mycobacterial and viral infections. The non-specific effects of BCG occur via the induction of trained immunity and form the basis for the hypothesis that BCG vaccination could be used to protect against the severity of coronavirus disease 2019 (COVID-19). This Perspective article highlights key milestones in the 100-year history of BCG and projects its potential role in the COVID-19 pandemic.

The Bacillus Calmette-Guérin (BCG) vaccine has been used since 1921 to prevent tuberculosis (TB) and is considered to be the world's most widely used vaccine ${ }^{1}$. In 2002, an estimated $75 \%$ of the 130 million children born worldwide were vaccinated with BCG $^{2}$. BCG is also an established anti-cancer immunotherapy and is used intravesically for intermediate-risk and high-risk non-muscle-invasive bladder cancer (NMIBC) to prevent recurrence and progression ${ }^{3}$.

The beginning of 2020 saw the outbreak of the coronavirus disease 2019 (COVID-19) pandemic caused by severe acute respiratory syndrome coronavirus 2 (SARS-CoV-2) ${ }^{4}$. With a global death toll of more than 3 million at the time of writing (7 May 2021), the pandemic has placed extraordinary demands on health-care systems ${ }^{5}$. A growing body of evidence suggests that the benefits of BCG extend beyond uses in TB and bladder cancer, with vaccination offering additional protection against various unrelated pathogens, including those that cause non-mycobacterial and viral infections $s^{6-15}$. Indeed, emerging data suggest that these non-specific effects might be protective against COVID-19 $\left(\mathrm{REFS}^{16-20}\right)$, and several randomized controlled trials are underway to investigate this hypothesis (TABLE 1). Thus, the COVID-19 pandemic provides a timely opportunity for urologists to reflect on the past 100 years of BCG in clinical practice.

In this timeline Perspective, we review the origins of BCG, its mechanisms of action and contemporary evidence for its use in $\mathrm{TB}$, non-mycobacterial diseases and bladder cancer. We also discuss the role and use of BCG in the COVID-19 era and implications of this use for the urological community.

\section{Origins of BCG}

Following the discovery of Mycobacterium tuberculosis as the causative organism in TB by Robert Koch in 1882, scientists raced to develop a vaccine as the TB epidemic peaked across industrialized European cities (FIC. 1). In 1908, Albert Calmette and Camille Guérin began working to develop an effective vaccination against $\mathrm{TB}$ at the Pasteur Institute in Lille, France. Using 'lait Nocard', a virulent strain of Mycobacterium bovis originating from a cow with tuberculous mastitis, the pair subcultured the organism every 3 weeks on a glycerinated beef-bile-potato medium. After 13 years and more than 200 passages, the organism was finally attenuated to a weakened non-virulent form. Calmette and Guérin gave their names to this strain of $M$. bovis and the resulting vaccine became known as BCG ${ }^{21}$.

In 1921, Benjamin Weill-Halle and Raymond Turpin orally administered the first $B C G$ vaccine to a neonate whose mother had died from TB at the Charité Hospital in Paris. The baby experienced no side effects from the vaccine and did not develop TB, despite maternal exposure. Over the next 3 years, 217 Parisian children were successfully vaccinated against $\mathrm{TB}$, and mass production of the BCG vaccine commenced at the Pasteur Institute. The League of Nations declared the vaccine safe for use in 1928 and vaccination programmes continued uneventfully until 1930, when what would later be known as the Lübeck disaster occurred, in which 72 neonates in Lübeck, Germany, died after receiving the BCG vaccination ${ }^{22}$. Although their deaths were later shown to be due to accidental contamination of vaccine preparations with a virulent strain of M. tuberculosis, BCG vaccination suffered a major setback and enthusiasm for its use rapidly waned. However, a resurgence of TB during World War II led to a return of widespread BCG vaccination and public trust in its safety was restored ${ }^{23}$.

In 1974, the WHO created the Expanded Programme on Immunization to ensure universal access of mothers and children to routinely recommended infant vaccines. Intradermal (ID) BCG vaccination at birth has been included in this programme since inception, resulting in more than 4 billion vaccines administered to date ${ }^{24}$.

\section{BCG strains}

$\mathrm{BCG}$ is no longer a single vaccine (FIG. 2). Calmette and Guérin's original strain was distributed to several laboratories across the world, and a number of diverse daughter strains have evolved as a result of genetic variation during decades of in vitro passage ${ }^{25}$. The main genetic modification involved in the attenuation of the strain is the loss of the genomic region of difference 1 (RD1), which generated the earliest BCG substrains, including BCG Japan, Birkhaug, Sweden, Russia and Moreau. Subsequently, 
Table 1 | Summary of randomized controlled trials of BCG vaccination and COVID-19

\begin{tabular}{|c|c|c|c|c|c|}
\hline Study & Location & Primary outcome & $\begin{array}{l}\text { Estimated } \\
\text { enrolment }\end{array}$ & Status & $\begin{array}{l}\text { Projected end } \\
\text { date }\end{array}$ \\
\hline $\begin{array}{l}\text { Application of BCG vaccine for } \\
\text { immune-prophylaxis among Egyptian } \\
\text { health-care workers during the pandemic } \\
\text { of COVID-19 } \\
\text { (NCT04350931) }^{166}\end{array}$ & Egypt & Incidence of COVID-19 infection & 900 & $\begin{array}{l}\text { Not yet } \\
\text { recruiting }\end{array}$ & December 2020 \\
\hline $\begin{array}{l}\text { Efficacy of BCG vaccination in the } \\
\text { prevention of COVID-19 via the } \\
\text { strengthening of innate immunity in } \\
\text { health-care workers (COVID-BCG) } \\
\text { (NCT04384549) }^{167}\end{array}$ & France & $\begin{array}{l}\text { Incidence of documented COVID-19 } \\
\text { among health-care workers exposed to } \\
\text { SARS-CoV-2 and vaccinated with BCG } \\
\text { compared with placebo }\end{array}$ & 1,120 & Recruiting & February 2021 \\
\hline $\begin{array}{l}\text { Reducing health-care workers } \\
\text { absenteeism in the COVID-19 pandemic } \\
\text { through the BCG vaccine (BCG-CORONA) } \\
\text { (NCT04328441) }^{168}\end{array}$ & Netherlands & $\begin{array}{l}\text { Number of days of health-care workers' } \\
\text { unplanned absenteeism for any reason }\end{array}$ & 1,500 & $\begin{array}{l}\text { Active, not } \\
\text { recruiting }\end{array}$ & April 2021 \\
\hline $\begin{array}{l}\text { Prevention of respiratory tract infection } \\
\text { and COVID-19 through BCG vaccination } \\
\text { in vulnerable older adults (BCG-PRIME) } \\
\text { (NCT04537663) }^{170}\end{array}$ & Netherlands & $\begin{array}{l}\text { Incidence of clinically relevant } \\
\text { respiratory tract infection or COVID-19 } \\
\text { infection }\end{array}$ & 5,200 & Recruiting & April 2021 \\
\hline $\begin{array}{l}\text { Clinical trial evaluating the effect of } \\
\text { BCG vaccination on the incidence and } \\
\text { severity of SARS-CoV-2 infections among } \\
\text { health-care professionals during the } \\
\text { COVID-19 pandemic in Poland } \\
(\text { NCT04648800) }\end{array}$ & Poland & Incidence of COVID-19 infections & 1,000 & Recruiting & April 2021 \\
\hline $\begin{array}{l}\text { BCG vaccination to prevent COVID-19 } \\
\text { (ACTIVATEII) } \\
(\text { NCT04414267) }\end{array}$ & Greece & $\begin{array}{l}\text { Positive for the respiratory questionnaire } \\
\text { consisting of questions concerning } \\
\text { the appearance of symptoms possibly, } \\
\text { probably and/or definitively related to } \\
\text { COVID-19 on visit } 3\end{array}$ & 900 & Recruiting & May 2021 \\
\hline $\begin{array}{l}\text { Reducing COVID-19-related hospital } \\
\text { admission in the elderly by BCG } \\
\text { vaccination } \\
(\text { NCT04417335) }\end{array}$ & Netherlands & SARS-CoV-2 hospital admission & $2,014^{\star}$ & $\begin{array}{l}\text { Active, not } \\
\text { recruiting }\end{array}$ & May 2021 \\
\hline $\begin{array}{l}\text { Study to assess VPM1002 in reducing } \\
\text { hospital admissions and/or severe } \\
\text { respiratory infectious diseases in the } \\
\text { elderly in the COVID-19 pandemic } \\
\text { (NCT04435379) }^{133}\end{array}$ & Germany & $\begin{array}{l}\text { Number of days with severe respiratory } \\
\text { disease at hospital and/or at home }\end{array}$ & 2,038 & $\begin{array}{l}\text { Active, not } \\
\text { recruiting }\end{array}$ & September 2021 \\
\hline $\begin{array}{l}\text { Efficacy and safety of VPM1002 in } \\
\text { reducing SARS-CoV-2 (COVID-19) } \\
\text { infection rate and severity (COBRA) } \\
(\mathrm{NCT} 44439045)^{132}\end{array}$ & Canada & Incidence of COVID-19 infection & 3,626 & $\begin{array}{l}\text { Active, not } \\
\text { recruiting }\end{array}$ & December 2021 \\
\hline $\begin{array}{l}\text { Using BCG vaccine to protect health-care } \\
\text { workers in the COVID-19 pandemic } \\
(\text { NCT04373291) }\end{array}$ & Denmark & $\begin{array}{l}\text { Days of unplanned absenteeism for any } \\
\text { reason }\end{array}$ & 1,500 & $\begin{array}{l}\text { Active, not } \\
\text { recruiting }\end{array}$ & December 2021 \\
\hline $\begin{array}{l}\text { BCG to reduce absenteeism among } \\
\text { health-care workers during the COVID-19 } \\
\text { pandemic (EDCTP) } \\
\left(^{(N C T 04641858)^{175}}\right.\end{array}$ & Denmark & $\begin{array}{l}\text { Days of unplanned absenteeism due to } \\
\text { illness }\end{array}$ & 1,050 & Recruiting & March 2022 \\
\hline $\begin{array}{l}\text { Using BCG to protect senior citizens } \\
\text { during the COVID-19 pandemic } \\
(\text { NCT04542330) }\end{array}$ & Denmark & Incidence of acute infection & 1,900 & Recruiting & March 2022 \\
\hline
\end{tabular}


Table 1 (cont.) | Summary of randomized controlled trials of BCG vaccination and COVID-19

\begin{tabular}{|c|c|c|c|c|c|}
\hline Study & Location & Primary outcome & $\begin{array}{l}\text { Estimated } \\
\text { enrolment }\end{array}$ & Status & $\begin{array}{l}\text { Projected end } \\
\text { date }\end{array}$ \\
\hline $\begin{array}{l}\text { Use of BCG vaccine as a preventive } \\
\text { measure for COVID-19 in health-care } \\
\text { workers (ProBCG) } \\
\text { (NCT04659941) }^{177}\end{array}$ & Brazil & $\begin{array}{l}\text { Incidence of SARS-CoV-2 infection; } \\
\text { incidence of severe forms of COVID-19 }\end{array}$ & 1,000 & Recruiting & October 2022 \\
\hline 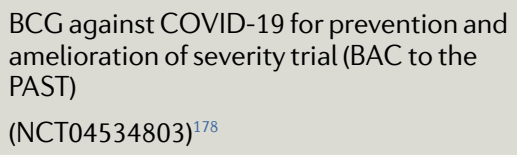 & USA & Incidence of severe COVID-19 disease & 2,100 & $\begin{array}{l}\text { Not yet } \\
\text { recruiting }\end{array}$ & November 2022 \\
\hline $\begin{array}{l}\text { COVID-19: BCG as therapeutic } \\
\text { vaccine, transmission limitation and } \\
\text { immunoglobulin enhancement (BATTLE) } \\
\text { (NCT04369794) }^{179}\end{array}$ & Brazil & $\begin{array}{l}\text { Clinical evolution of COVID-19; } \\
\text { SARS-CoV-2 elimination; seroconversion } \\
\text { rate and titration (lgA, IgM, IgG) }\end{array}$ & 1,000 & Recruiting & August 2023 \\
\hline
\end{tabular}

deletion of RD2 led to the late strains, including BCG Pasteur, Danish, Glaxo, Tice, Montreal (also known as Frappier) and Connaught. Although $>20$ genetically distinct BCG strains exist ${ }^{26}, 5$ strains currently account for $>90 \%$ of the vaccines used worldwide: Pasteur 1173 P2, Danish 1331, Glaxo 1077 (derived from the Danish strain), Tokyo 172-1 and Russian BCG-I ${ }^{27}$. Other commonly used strains include the Montreal and Tice strains, the latter of which is the only strain available for intravesical use in patients with NMIBC in the USA following suspension of BCG-Connaught production by the FDA in 2011 after mould was discovered at the manufacturing facility. As the Connaught strain supplied a significant proportion of the global market, the end of its production has led to a worldwide BCG shortage ${ }^{28}$.

Observational studies suggest that various BCG vaccine strains might have different immunogenicity and adverse reaction profiles, with the Russian strain potentially associated with reduced scarification $^{29-33}$. A 2019 randomized controlled trial comparing vaccination with the BCG-Denmark (Copenhagen 1331), BCG-Japan (Tokyo 172-1) and BCG-Russia (Russia BCG-I) strains in neonates in Guinea-Bissau found no difference in all-cause morbidity (BCG-Denmark versus BCG-Russia, hospitalization incidence rate ratio (IRR) 1.08 (95\% CI 0.84-1.37); BCG-Japan versus BCG-Russia, IRR 1.15 (95\% CI 0.93-1.43)) or mortality (BCG-Denmark versus BCG-Russia, mortality rate ratio 1.15 (95\% CI 0.74-1.80); BCG-Japan versus BCG-Russia, mortality rate ratio 0.71 (95\% CI 0.43-1.19)) among strains $^{34}$. However, in keeping with other studies, BCG-Russia produced fewer scars than other strains (BCG-Denmark versus BCG-Russia, prevalence ratio (PR) 1.07
(95\% CI 1.04-1.10); BCG-Japan versus BCG-Russia, PR 1.06 (95\% CI 1.02-1.09)). Although the presence of a BCG vaccine scar does not predict susceptibility to $\mathrm{TB}^{26}$, these findings suggest that differences could exist in the immune response to different BCG strains. Further evidence supporting this hypothesis comes from a large randomized trial in 303,092 neonates in Hong Kong that showed that the risk of TB after vaccination with BCG-Pasteur was $45 \%$ (95\% CI $22 \%-61 \%)$ less than the risk after vaccination with BCG-Glaxo ${ }^{35}$. Additionally, a cohort study in Kazakhstan showed differences between the efficacy of different strains: neonatal vaccination with BCG-Tokyo, BCG-Serbia and BCG-Russia was shown to reduce the risk of clinically diagnosed TB by $69 \%$ (95\% CI 61-75\%), $43 \%$ (95\% CI 31-53\%) and 22\% (95\% CI 7-35\%), respectively ${ }^{36}$.

Whether strain selection influences the efficacy of intravesical BCG in treating NMIBC is unclear. Several different strains including BCG Tice, BCG Moreau, BCG Connaught and BCG Tokyo-172 with different quantities of colony-forming units are currently in use for intravesical instillations ${ }^{37}$. In a prospective randomized controlled trial comparing BCG Connaught with BCG Tice in 132 patients with high-risk NMIBC, 5-year recurrence-free survival was significantly higher in the BCG Connaught group (74\% versus $48 \%, P=0.0108$ ), but progression-free survival $(P=0.3442)$ and overall survival $(P=0.2652)$ did not differ between the groups ${ }^{38}$. However, this study had several limitations, including a lack of maintenance treatment. A larger retrospective study comparing the same strains in 2,099 patients showed that BCG Connaught was more effective than BCG Tice for time-to-first recurrence (HR 1.48; 95\% CI 1.20-1.82; $P<0.001)^{39}$.
However, when maintenance BCG was used, the opposite was true (HR 0.66; $95 \%$ CI $0.47-0.93, P=0.019)$. The authors hypothesize that this might be due to BCG Connaught reaching an earlier cumulative optimized dose than BCG Tice $^{39}$. Subsequently, a 2020 retrospective comparison of BCG Tice $(n=321)$ with BCG Moreau $(n=339)$ showed no difference in recurrence-free (HR 0.88; 95\% CI 0.56-1.38, $P=0.58$ ) or progression-free survival (HR 0.55 ; 95\% CI $0.25-1.21, P=0.14)^{40}$. Likewise, a meta-analysis comparing nine strains across 65 trials was unable to demonstrate the superiority of one BCG strain over another at preventing recurrence ${ }^{41}$. Ultimately, comparative trials are needed to determine the effect of strain selection on BCG efficacy and to identify whether an optimal strain exists for treatment of NMIBC.

\section{BCG and tuberculosis}

TB is the leading infectious cause of death globally, responsible for an estimated 1.4 million deaths in 2019 (REF. ${ }^{42}$ ).

In countries with a high incidence of TB ( $\geq 40$ per 100000 ), BCG vaccination reduces the risk of childhood meningeal and miliary TB by $85 \%{ }^{43}$ and pulmonary TB by $50 \%{ }^{44}$. These protective effects last $\sim 10-15$ years, although a small number of studies have demonstrated protection of up to 50 years after vaccination ${ }^{45,46}$.

However, adults and adolescents, not children, are thought to be responsible for the majority of community transmission of $\mathrm{TB}^{47}$. The protective effect of BCG vaccination is far more variable in adolescents and adults than children, with protection rates ranging from $0-80 \%{ }^{48}$. Reasons for this high variability are not clear, but could include differences in BCG strain, host genetics and previous exposure to environmental mycobacteria that can 


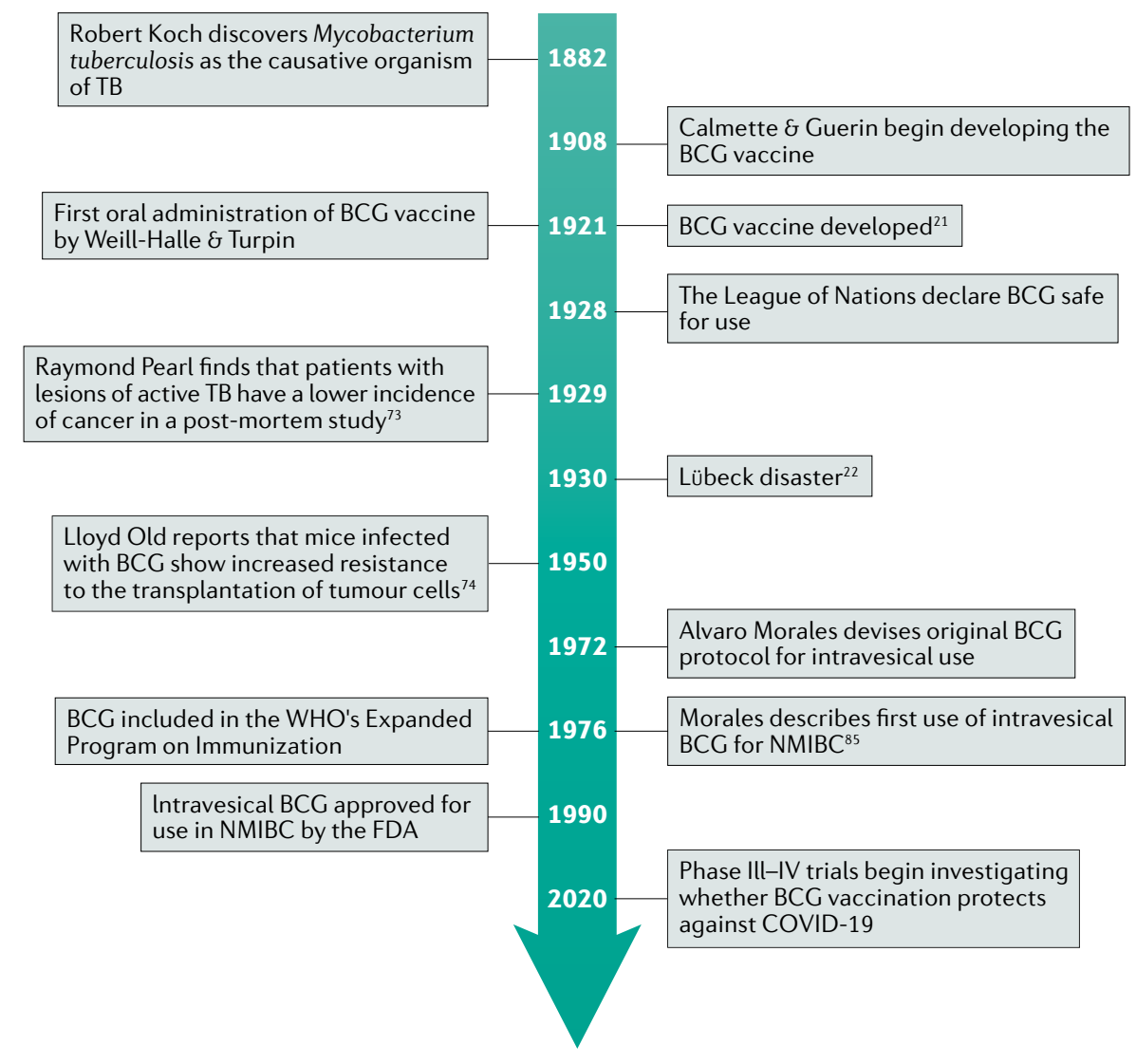

Fig. 1 | The history of Bacillus Calmette-Guérin (BCG) from discovery to use in COVID-19. FDA, US Food and Drug Administration; NMIBC, non-muscle-invasive bladder cancer; TB, tuberculosis.

confer some underlying protection against M. tuberculosis ${ }^{49,50}$.

A vaccine that prevents pulmonary $\mathrm{TB}$ in adolescents and adults is, therefore, urgently needed for TB control. Fortunately, novel insights into the basic immunology of $M$. tuberculosis infection and clinical vaccine development have led to the development of potential vaccine candidates, some of which are now in advanced clinical trials. In particular, two phase IIb trials are worth highlighting. In a 2018 clinical trial of re-vaccination of adolescents in South Africa, a reduction in the rate of TB infection was observed in the BCG re-vaccinated groups (efficacy $45.4 \%, P=0.03$ ), as measured by the rate of sustained Quantiferon-TB Gold In-tube assay conversion, compared with participants who received a placebo vaccination $(30.5 \%$, $P=0.16)^{51}$. In a second 2018 trial, protection against TB could be achieved by vaccination of $M$. tuberculosis-infected adults with an adjuvanted subunit vaccine containing two M. tuberculosis proteins (efficacy 54\%, 95\% CI $13.9-75.4 \% ; P=0.04)^{52}$.

Interestingly, although intravenous (IV) BCG injections had been consigned to the history books, this approach was revisited in a 2020 study in adult rhesus macaques ${ }^{53}$. The study compared ID and IV injection of BCG and observed that IV BCG was highly effective in protecting against M. tuberculosis challenge, with six out of ten vaccinated animals showing no signs of infection as defined by lack of granuloma formation. Furthermore, nine out of ten IV BCG-immunized macaques had no signs of tuberculous disease, as determined by a lack of lung fluorodeoxyglucose activity at the time of necropsy. By contrast, no evidence of protection against infection or disease was seen in macaques receiving ID injection of the standard human dose. Although these results are encouraging, meticulous safety testing is required to prove that IV administration is safe in humans. As seen in the Lübeck disaster, direct IV injection of a contaminated vaccine could lead to fatal infection. Nevertheless, these studies represent milestones in the efforts to develop a novel TB vaccine that protects against both neonatal and adult TB.

\section{Non-specific effects of BCG}

A growing body of evidence indicates that vaccines have non-specific effects on the immune system ${ }^{54}$. Also known as heterologous or off-target effects, these afford additional protection beyond that offered against their target disease.

Over the past 50 years, numerous studies have proved that BCG vaccination is able to induce non-specific protective effects. Carl Naslund in Sweden first described this phenomenon in 1932, noting that BCG-vaccinated neonates $(n=1,323)$ had a mortality rate that was almost threefold lower than those who were unvaccinated $(n=3,350 ; 6.6 \% \text { versus } 22.2 \%)^{55}$. Subsequent epidemiological studies have corroborated this finding, showing a correlation between BCG vaccination and reduced childhood mortality ${ }^{56-58}$, and hospitalization rates attributable to other respiratory infections $s^{59}$, independent of its effect on TB.

Strong evidence for the non-specific effects of BCG vaccination comes from a series of randomized controlled trials in Guinea-Bissau, a country with a high childhood mortality rate $\mathrm{e}^{58,60-63}$. The main study found that low-birth-weight neonates vaccinated at birth had a $>40 \%$ reduction in neonatal mortality rate compared with low-birth-weight neonates who had delayed vaccination at 6 weeks ${ }^{62}$. This effect seems to be mainly due to protection against neonatal sepsis and respiratory infection ${ }^{61}$. Subsequently, the phase III ACTIVATE trial assessed whether BCG confers similar protective effects against respiratory infections in the elderly ${ }^{15}$. In this double-blind, randomized trial, 198 hospitalized elderly patients received either BCG or placebo vaccine at discharge and were followed up for 12 months for the occurrence of new infections. At interim analysis, BCG vaccination significantly increased the time to first infection compared with placebo (median 16 weeks versus 11 weeks; $P=0.039$ ). The incidence of new infections was $42.3 \%$ (95\% CI 31.9\%-53.4\%) after placebo vaccination compared with $25 \%$ (95\% CI $16.4 \%-36.1 \%$ ) after BCG vaccination; most of the protection in the BCG group was determined to be against respiratory tract infection of probable viral origin (HR 0.21, $P=0.013$ ).

Evidence from murine models and human trials has shown that BCG vaccination protects against secondary infections with Candida albicans ${ }^{6,7}$, Schistosoma mansoni $i^{8}$ and Plasmodium ${ }^{9-12}$. In a randomized study, participants received either BCG or placebo vaccination followed by a trivalent seasonal influenza vaccine 14 days later ${ }^{13}$. The data showed that BCG-vaccinated subjects had markedly enhanced antibody responses against the 
A(H1N1)pdm09 vaccine strain compared with patients who had received placebo (0.041), with a trend towards more rapid seroconversion $(P=0.08)$. In another randomized placebo-controlled study, yellow fever vaccine recipients who had received the BCG vaccine 1 month previously had significantly lower yellow fever vaccine viraemia than subjects who had received previous placebo vaccination $(P<0.05)^{14}$. The lower vaccine viraemia was also accompanied by lower circulating inflammatory cytokines in BCG-vaccinated individuals - lower levels of circulating inflammatory cytokines were seen because the high cytokine production capacity following BCG vaccination leads to a rapid local antimicrobial response and subsequent elimination of the pathogen, thereby preventing a systemic reaction and high levels of circulating cytokines ${ }^{64,65}$. However, yellow fever antibody titres did not differ between placebo and BCG-vaccinated groups 90 days after receiving the vaccine, indicating that the trained immunity induced by BCG confers protection via an antigen-unspecific process ${ }^{66}$.

BCG vaccination has also been shown to have a protective effect against atopy measured by skin-test reactivity to three allergens, Dermatophagoides pteronyssinus, Dermatophagoides farinae and cockroach ${ }^{67}$. This finding has been validated in randomized controlled trials; one Dutch study of 121 newborns showed that BCG-vaccinated infants used significantly less medication for eczema than unvaccinated infants $(P=0.04)^{68}$. In the separate Dutch Calmette Study, neonatal BCG vaccination reduced the cumulative incidence of atopic dermatitis in children with atopic predisposition compared with BCG-unvaccinated predisposed infants (22.7\% versus $25.4 \%$; RR 0.90 (95\% CI $0.8-1.0))^{69}$. Furthermore, in a clinical trial of BCG vaccination with a 60 -year follow-up period, BCG-vaccinated children had a significantly reduced risk of lung cancer development than those who received placebo (18.2 versus 45.4 cases per 100,000 person-years; 95\% CI 0.20-0.74, $P=0.05$ ), even when controlling for other risk factors including smoking, sex, region and alcohol overuse $^{70}$. Notably, however, the overall rate of cancer diagnosis was not significantly different in BCG vaccine versus placebo recipients (HR 0.82; 95\% CI 0.66-1.02).

\section{BCG and cancer}

The concept of using microbial products as immunotherapy for cancer was pioneered by William B. Coley at the turn of the 20th Century. After learning about a patient with an inoperable malignant neck tumour that disappeared after developing erysipelas, Coley started treating his own patients with cancer with intratumoural injections of live Streptococcus pyogenes ${ }^{71}$ and, later, mixtures of S. pyogenes and Serratia marcescens ${ }^{72}$. Although he observed tumour regression in several patients, the injections had unpredictable efficacy and lethal adverse effects sometimes occurred.

In 1929, Raymond Pearl noted that patients with lesions of active TB had a lower frequency of cancer on post-mortem examination $^{73}$, although he was unable to provide an explanation for this finding. In 1959, landmark studies by Lloyd Old at the Memorial Sloan-Kettering Institute in New York demonstrated the inhibition of cancer by BCG: mice infected with BCG showed increased resistance to the transplantation of the murine tumour cell lines S-180, carcinoma 755 and Ehrlich ascites $^{74}$.

Further work published in 1971 by Zbar and Tanaka at the National Cancer Institute was instrumental in confirming the anti-neoplastic properties of $\mathrm{BCG}^{75}$. $\mathrm{Zbar}$ found that intralesional injection of BCG into guinea pigs induced regression of ID tumours, prevented the development of lymph node metastases and eradicated nodal micrometastases ${ }^{75}$. Zbar later went on to describe the four criteria for optimal BCG therapy: proximity between BCG and tumour cells, a small tumour burden, a host capable of mounting an immunological reaction to mycobacterial antigens, and sufficient numbers of viable mycobacteria ${ }^{76}$. This work led to the clinical use of BCG as cancer therapy, first in acute lymphoblastic leukaemia, in which adjuvant BCG reduced relapse rates $^{77}$, and later in malignant melanoma in which skin lesions regressed following treatment with intralesional BCG injection ${ }^{78}$. These reports created a great deal of interest in BCG and prompted a number of clinical trials using BCG against kidney ${ }^{79}$, colon ${ }^{80}$ and lung $^{81}$ cancer. Unfortunately, these trials failed to show a significant benefit of BCG in these tumour types, although, notably, most of the studies were in patients with advanced disease who, based on Zbar's criteria, would not be expected to respond to $\mathrm{BCG}^{82}$.

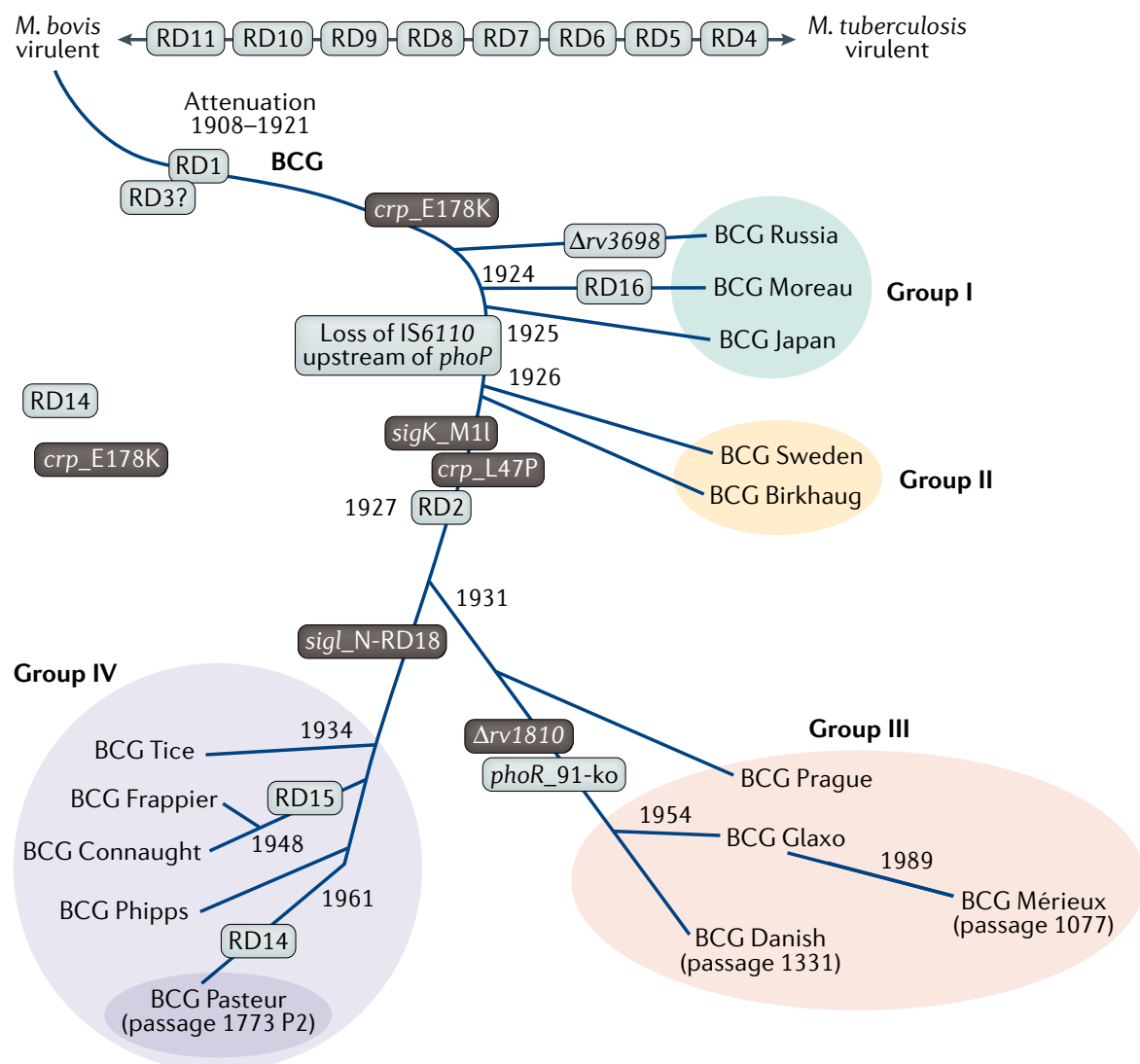

Fig. 2 | Historical phylogeny of BCG vaccine strains. Continuous passage of the original strain attenuated by Calmette and Guérin resulted in a number of diverse daughter strains. Loss of the genomic region of difference 1 (RD1) generated the early Bacillus Calmette-Guérin (BCG) strains (groups I and II). Late strains (groups III and IV) were subsequently generated by deletion of RD2. Adapted from Brosch et al. ${ }^{25}$, PNAS. Copyright (2007) National Academy of Sciences, USA. 


\section{BCG and bladder cancer}

The most successful application of BCG for the treatment of malignancy was in NMIBC. Preliminary work by Coe and Feldman showed that BCG elicited a delayed hypersensitivity response in guinea pig bladders ${ }^{83}$, and when injected into a malignant melanoma metastasis in the bladder, complete eradication was observed ${ }^{84}$.

In the early 1970s, Alvaro Morales started investigating the use of BCG in bladder cancer and in 1972 devised the BCG protocol that remains in use today ${ }^{85}$. Interestingly, Morales chose the schedule of six weekly instillations simply because the Armand Frappier strain of BCG he was using was supplied in six separate vials with adverse events lasting less than 1 week $^{85}$. In a landmark study in 1976, Morales treated nine patients with recurrent NMIBC with weekly intravesical and ID BCG for 6 weeks, observing a 12 -fold reduction in bladder cancer recurrence $(P<0.01)^{85}$. These promising data prompted two randomized controlled trials testing the effectiveness of transurethral resection (TUR) followed by intravesical BCG for NMIBC versus surgical resection alone. In a 1980 study in 37 patients, BCG significantly reduced tumour recurrence rate compared with resection alone ( $17 \%$ versus $42 \%$, $P=0.029)^{86}$. Similarly, a 1985 randomized trial of 86 patients with 43 in each treatment arm demonstrated a significant reduction in the number of recurrent tumours in patients receiving BCG compared with a control group receiving standard care (cystoscopy and fulguration) (43 versus 27 patients, respectively; $P<0.001)^{87}$.

Furthermore, patients treated with BCG also demonstrated reduced tumour progression requiring cystectomy compared with patients in the control group ( 3 versus 15 patients, respectively, $P<0.001)^{87}$. Subsequent meta-analyses have confirmed that BCG is associated with a decreased risk of recurrence compared with TUR alone ${ }^{88,89}$. Han et al. analysed 4,767 patients undergoing TUR from 25 randomized controlled trials and found a significant difference in the odds ratio for tumour recurrence between groups treated with and without BCG (OR 0.61, 95\% CI 0.46-0.80, $P<0.0001)^{89}$. BCG has also been shown to be superior to chemotherapy for recurrence prevention $^{90-92}$. A meta-analysis of 2,749 patients from 11 randomized controlled trials reported that BCG was superior to mitomycin $\mathrm{C}$ in reducing tumour recurrence (OR 0.56, 95\% CI 0.38-0.84, $P=0.005)^{90}$. Furthermore, in the subgroup treated with BCG maintenance, all six studies showed significant superiority of BCG over mitomycin C (OR 0.43, 95\% CI 0.35-0.53, $P<0.001)^{90}$. Similarly, in a meta-analysis using individual patient data from nine randomized controlled trials $(n=2,820)$, Malmstrom and colleagues observed a 32\% reduction in risk of recurrence in patients treated with maintenance BCG compared with those treated with mitomycin $\mathrm{C}$ $(P<0.0001)^{92}$. Patients treated without maintenance BCG demonstrated a $28 \%$ increase in recurrence risk $(P=0.006)^{92}$.

With regard to tumour progression, several meta-analyses have concluded that intravesical BCG prevents - or at least delays - progression to invasive disease ${ }^{93,94}$. The largest of these studies analysed 24 trials ( $n=4,863$ patients) and showed a $27 \%$ reduction in the odds of progression (9.8\% versus $13.8 \%$; OR $0.73, P=0.001)$ in patients treated with maintenance BCG compared with either TUR alone or TUR with chemotherapeutic agents other than mitomycin $\mathrm{C}^{93}$. Notably, however, the Malmstrom meta-analysis was unable to confirm a significant difference between $\mathrm{BCG}$ and mitomycin $\mathrm{C}$ for either progression $(P=0.6164)$ or survival $(P=0.2783)^{92}$. These conflicting results could perhaps be explained by differences in patient characteristics, maintenance schedules, follow-up period and statistical power ${ }^{95}$.

Another area of controversy related to BCG therapy is the optimal duration of maintenance regimens. Although different regimens have been used, a 2002 meta-analysis was unable to determine which regimen was most effective for NMIBC treatment ${ }^{93}$. However, a 3-year maintenance schedule is supported by data from the SWOG 8507 (REF. ${ }^{96}$ ) and EORTC 30962 (REF. ${ }^{97}$ ) studies. SWOG 8507 showed that maintenance BCG given as a weekly instillation for 3 weeks over a 3 -year period increased the 5-year recurrence-free survival from $41 \%$ to $60 \%(P<0.0001)$ compared with induction $\mathrm{BCG}$ alone ${ }^{96}$. Accordingly, EORTC 30962 used a similar regimen and showed that 3 years of maintenance treatment reduced the recurrence rate compared with 1 year of BCG maintenance in patients with high-risk disease $(95 \% \mathrm{CI}$ 1.13-2.30; $P=0.009)^{97}$. As such, current European Association of Urology (EAU) and American Association of Urology(AUA)/ Society of Urologic Oncology (SUO) guidelines recommend a risk-stratified schedule for maintenance therapy, such that patients with intermediate-risk disease should receive maintenance therapy for 1 year whereas patients with high-risk disease receive treatment for up to 3 years ${ }^{3,98}$.
Despite high success rates, BCG fails in approximately $25-45 \%$ of patients, with a further $40 \%$ of patients eventually relapsing despite an initial response $\mathrm{e}^{9699-101}$. Furthermore, intravesical BCG can cause adverse effects, which - although mild in the majority of cases - can be poorly tolerated and curtail treatment schedules ${ }^{102}$. Haematuria and cystitis are the most common local side effects occurring in approximately $20 \%$ and $35 \%$, respectively, and tend to arise in the first $48 \mathrm{~h}$ after BCG instillation ${ }^{103}$. Systemic adverse effects include pyrexia, fatigue and general malaise. Severe systemic effects are rare $(<1 \%)$ and are the result of disseminated infection (also known as BCG-osis), which can present as sepsis, reactive arthritis, pneumonitis, rash, or - rarely - mycotic aneurysm ${ }^{104-106}$.

\section{Mechanisms of BCG in bladder cancer}

The precise mechanism of action by which BCG immunotherapy exerts its effect remains incompletely elucidated. However, it is clear that BCG induces a robust immune response that leads to adaptive immunity and anti-tumour activity. Following intravesical instillation, BCG is believed to attach to and invade the urothelium. Although murine studies indicate that BCG binds to the urothelium via fibronectin receptors ${ }^{107}$, this mechanism of attachment is not supported by studies in humans ${ }^{108,109}$. Regardless, once BCG attaches to the urothelium, it is internalized by urothelial cells, bladder cancer cells and immune cells ${ }^{110}$. Internalization stimulates a local and systemic innate immune response leading to the release of several chemokines and cytokines including IL-6, IL-8, GM-CSF and TNF ${ }^{111-113}$. In fact, the release of these pro-inflammatory cytokines might be used to predict response to BCG therapy for bladder cancer. For example, the CyPRIT assay, a nomogram constructed using urinary levels of cytokines induced by BCG (IL-2, IL-6, IL-8, IL-18, IL-1RA, TRAIL, IFN $\gamma$, IL-12p70 and TNF), was able to predict the likelihood of recurrence with $85.5 \%$ accuracy $(95 \%$ CI 77.9-93.1\% $)^{114}$. Following this cytokine and chemokine burst, cytotoxic immune cells, including neutrophils, monocytes, $\mathrm{CD}^{+}$ and $\mathrm{CD}^{+} \mathrm{T}$ cells, $\mathrm{B}$ cells, macrophages and natural killer (NK) cells, are recruited. Immune-mediated cytotoxicity of bladder cancer cells subsequently occurs via activation of NK cells, T cells and secretion of soluble factors such as TRAIL by polymorphonuclear cells ${ }^{110}$.

The success of BCG therapy also depends on the induction of adaptive 
immunity ${ }^{110}$. BCG antigens are presented on the cell surface of antigen-presenting cells and urothelial cells via MHC class II. These molecules interact with $\mathrm{CD} 4^{+}$ $\mathrm{T}$ cell receptors, leading to activation and differentiation of a primarily T helper 1 cell immune response $\mathrm{e}^{110,115}$. Together, both innate and adaptive immune system responses lead to lasting protection against tumour recurrence and progression ${ }^{110}$.

\section{BCG and trained immunity}

The non-specific effects of BCG might be explained in part by trained immunity - the concept that innate immune cells (such as monocytes and macrophages) encountering a vaccine or microbial component can be trained by a primary stimulus to improve responsiveness to a similar or unrelated secondary stimulus ${ }^{113}$.

Host immune responses are divided into innate and adaptive responses and, until relatively recently, it was believed that only adaptive immunity possessed immunological memory ${ }^{116}$. However, studies over the past decade have shown that innate immune cells also possess intrinsic memory characteristics ${ }^{7,14,117}$ and can undergo functional reprogramming to enable an enhanced response to secondary stimulation (FIG. 3). This reprogramming is achieved through histone modifications, which are epigenetic alterations of the chromatin structure in the nucleus that result in enhanced chromatin accessibility, and facilitate transcription of genes important for antimicrobial responses and improved cell function ${ }^{118}$. Additionally, metabolic changes such as increased glycolysis, oxidative phosphorylation and glucose consumption lead to selective accumulation or depletion of certain metabolites, such as glutamate and malate, that regulate this process ${ }^{119}$.

BCG vaccination is able to endow circulating monocytes with characteristics of trained immunity through epigenetic and metabolic rewiring of myeloid progenitors in the bone marrow ${ }^{120,121}$. This process begins with autophagy, the process of intracellular degradation of cell and microbial components. Phagosomal digestion of BCG causes the release of muramyl dipeptide (MDP) as well as metabolic changes through activation of the Akt-mTOR-HIF a pathway ${ }^{122}$. MDP binds to nucleotide-binding oligomerization domain-containing protein 2 (NOD2) receptor to induce epigenetic histone alterations ${ }^{7}$. Epigenetic modifications result in increased access to the promoter regions of genes related to inflammatory pathways, such as cytokines and pattern recognition

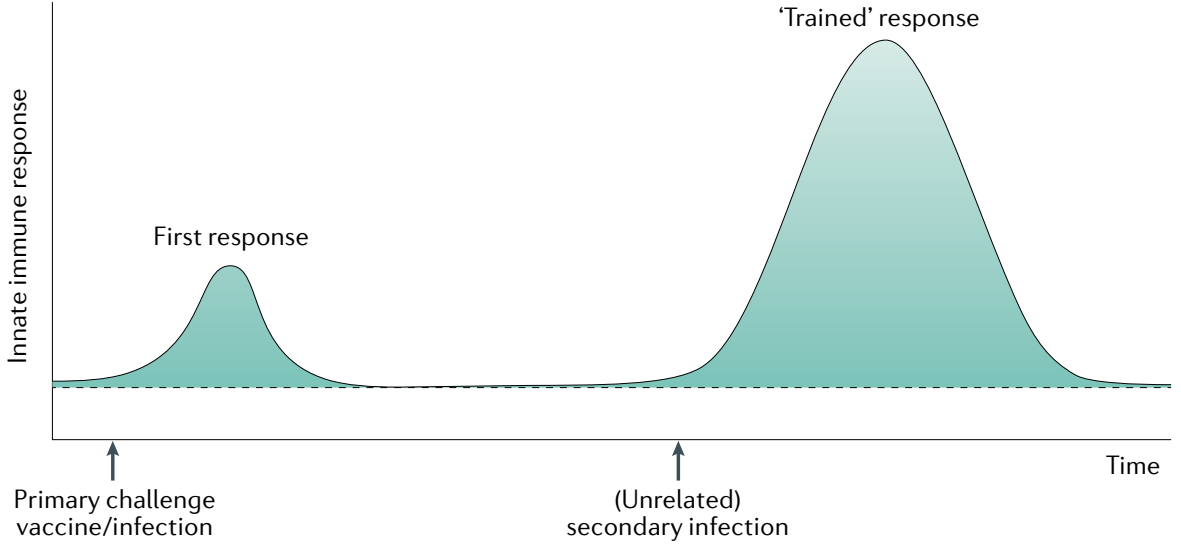

Fig. 3 | Proposed model of trained immunity. Innate immune responses during and after an infectious episode can lead to immunological programming, and a heightened immunity (trained immunity) that might translate into decreased susceptibility to (unrelated) secondary infections. Adapted from O'Neill and Netea ${ }^{180}$, Springer Nature Limited.

receptors. As a result, these BCG-trained innate immune cells express higher levels of pattern recognition receptors and produce increased levels of pro-inflammatory cytokines than non-BCG-trained cells when challenged by a secondary and potentially unrelated stimulus ${ }^{7,14}$, thus increasing the capacity of the innate immune cells to mount a faster and augmented response to such a secondary stimulation.

Emerging data have identified a key role for the stimulator of interferon genes (STING) pathway in mediating innate immune responses ${ }^{123,124}$. STING is a cytosolic receptor, and activation of STING induces potent cytokine and cellular immune responses against microbial infection ${ }^{123}$. STING-dependent signalling is activated by binding cyclic dinucleotides either secreted by intracellular bacteria or distinct host cyclic dinucleotides generated by host cell receptors after recognition of cytosolic double-stranded DNA ${ }^{125-127}$. STING agonists might, therefore, be a novel class of vaccine adjuvants capable of inducing cellular immune responses and protective efficacy against intracellular pathogens. Studies of a recombinant BCG that released high levels of STING agonist demonstrated a significantly augmented pro-inflammatory cytokine response (TNF, IL-6 $(P<0.05)$; IL-1 $\beta(P<0.001))$ in vitro in murine macrophages challenged with M. tuberculosis ${ }^{128}$. Attenuation of M. bovis for clinical use as BCG results in the loss of the early secretory antigenic target system (ESX-1), which is critical for activation of the STING pathway ${ }^{129}$. This loss potentially limits subsequent BCG responsiveness owing to reduced induction of immune cell-recruiting chemokines. To compensate, several recombinant versions of the BCG vaccine have been produced. One example is the VPM1002 vaccine, which activates the STING pathway and has been shown to be potentially more immunogenic than conventional BCG: in a phase II trial with a 6-month follow-up, 48 HIV-unexposed newborn infants were vaccinated with either VPM1002 $(n=36)$ or BCG Danish strain $(n=12)^{130}$. Although both vaccines induced IL-17 responses, VPM1002 vaccination led to a significant increase in $\mathrm{CD}^{+} \mathrm{IL}-17^{+} \mathrm{T}$ cells at day $14(P=0.0156)$ and month $6(P=0.0002)$ compared with $\mathrm{BCG}^{130}$. Trials are currently investigating whether VPM1002 can protect health-care workers $^{131,132}$ and the elderly ${ }^{133}$ against severe COVID-19 (TABLE 1).

\section{Trained immunity and bladder cancer}

Trained immunity might also have a role in the effect of intravesical BCG for NMIBC as an anti-tumour mechanism ${ }^{66}$. This role is not well defined; data on epigenetic modifications in innate immune cells during intravesical BCG instillations are lacking. However, increased cytokine ${ }^{134-136}$ and chemokine ${ }^{137,138}$ production by innate immune cells during intravesical BCG therapy is well described and might indirectly support a role for trained immunity in the mechanisms of BCG immunotherapy for NMIBC.

Further support for the role of trained immunity comes from studies evaluating the role of recombinant BCG and STING agonists in bladder cancer. The STING signalling pathway not only mediates innate immune responses against microbial infection but is also important for the anti-cancer immune response. Activation of the STING signalling pathway by tumour-derived DNA results in 
production of type I interferon and other pro-inflammatory cytokines that stimulate cross-presentation of tumour antigens and mobilization of tumour-specific $\mathrm{CD}^{+}$ $\mathrm{T}$ cells ${ }^{126}$. Thus, STING agonists have the potential to induce an anti-tumour immune response. Studies have evaluated the effect of BCG in combination with a STING agonist in cell-line models representing human monocytes, the bladder cancer cell line RT112 and primary bladder epithelial cells $^{139}$. They showed a $>20$-fold increase in immune cell recruiting chemokines including CXCL10 as a result of synergistic effect of BCG and the STING agonist ${ }^{139}$. In another study, use of a recombinant BCG strain overexpressing a STING agonist stimulated epigenetic changes that promoted enhanced anti-tumour immunity compared with wild-type BCG in rat NMIBC tumour models ${ }^{140}$. A significantly reduced number of immunosuppressive M2 macrophages and increased pro-inflammatory M1 macrophages $(P<0.01)$ was observed with the recombinant BCG strain compared with wild-type BCG. Additionally, myeloid cells treated with the recombinant BCG strain exhibited enhanced phagocytosis and autophagy to a greater degree than wild-type BCG. These findings highlight the improved ability of BCG to elicit trained immunity when combined with a STING agonist.

\section{BCG in the COVID-19 era}

The COVID-19 pandemic is a public health crisis caused by infection with SARS-CoV-2. COVID-19 has reached at least 212 countries and territories, with a global death toll of more than 3 million at the time of writing (7 May 2021) $)^{5}$. The outbreak of COVID-19 has placed extraordinary demands on health-care systems whilst also triggering a global recession that is set to exceed that of the 2008 financial crisis ${ }^{141,142}$.
Furthermore, the pandemic is predicted to last $\sim 2$ years, with further waves of infection occurring during winter or when quarantine regulations are relaxed ${ }^{143}$.

The pandemic has transformed cancer care, including that of patients with high-risk NMIBC. In particular, urologists must balance patients' risk of acquiring COVID-19 as a result of hospital visits for intravesical BCG instillations with the risks of recurrence and/or progression due to deferred treatment. Data show that people over the age of 65 years are at an increased risk of developing acute respiratory distress syndrome after contracting COVID-19 (HR 3.26 ; 95\% CI 2.08-5.11) ${ }^{144}$; the majority of patients with high-risk NMIBC fall into this age group and should, therefore, be regarded as an at-risk demographic ${ }^{145}$.

Several expert panels have produced recommendations for the use of intravesical BCG during the pandemic (FIG. 4). All agree that patients with high-risk NMIBC should receive induction $\mathrm{BCG}^{146,147}$ because the risk of recurrence and progression can be reduced by up to $70 \%$ and $26 \%$, respectively ${ }^{91,93}$. With regard to maintenance BCG, attempts should be made to provide patients with at least the first 3-week maintenance regimen and at least two out of the three doses of a maintenance course are acceptable in order to minimize the number of hospital visits ${ }^{147}$. For those patients who have completed more than a year of maintenance therapy, further doses can be safely discontinued ${ }^{148}$. When managing BCG-related adverse effects, urologists should have a low threshold for testing pyrexial patients for COVID-19 (REF. ${ }^{147}$ ).

\section{COVID-19 and BCG vaccination}

Given the non-specific protective effects of BCG vaccination against viral infection, these benefits have been hypothesized to

\section{EAU COVID guidelines 2020 (ref. ${ }^{146}$ )}

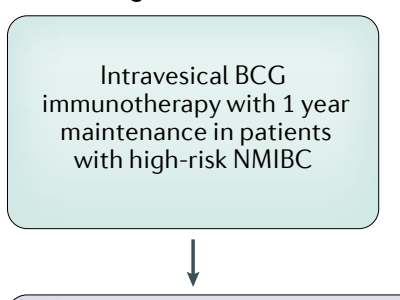

- Induction BCG should be given for high-risk NMIBC

- One year of maintenance BCG with at least two of three doses is acceptable for high-risk NMIBC - Patients who have completed $>1$ year of maintenance BCG do not require further doses

Fig. 4 | Summary of recommendations for intravesical BCG vaccine use in NMIBC in the COVID-19 era. BCG, Bacillus Calmette-Guérin; EAU, European Association of Urology; NMIBC, non-muscle-invasive bladder cancer. extend to protection against COVID-19 through the induction of trained immunity.

Ecological studies. Several ecological studies have proposed a correlation between BCG vaccination policies and COVID-19related mortality and morbidity ${ }^{16-19}$. For instance, countries with a BCG vaccination programme for TB had a lower daily incidence of COVID-19 (0.8 per million) than countries without such a programme $(34.8 \text { per million })^{16}$. Furthermore, both mortality and crude case fatality rate were lower in countries with a BCG vaccination programme compared with those without ( 0.08 per million and $4.1 \%$ versus 34.8 per million and $5.1 \%$ respectively). Similarly, the presence of mandatory national polices for universal BCG vaccination was associated with flattened growth curves for confirmed cases of COVID-19 and resulting deaths in the first 30-day period of country-wise outbreaks ${ }^{17}$. Overall, countries including BCG in their national vaccination programmes had a significantly lower incidence of COVID-19 cases (mean cases per population ratio: $0.0147 \pm 0.027$ versus $0.1892 \pm 0.244, P<0.0001)$ and mortality (mean of deaths per population ratio: $0.0004 \pm 0.001$ versus $0.0113 \pm 0.020$, $P<0.0001)$ than countries that excluded it ${ }^{18}$. Subsequently, an assessment of the global linkage between BCG vaccination and COVID-19 mortality ${ }^{19}$ showed a strong correlation between the BCG index (an estimation of the degree of universal BCG vaccination deployment in a country) and COVID-19 mortality $\left(\mathrm{r}^{2}=0.99, P=8 \times 10^{-7}\right)$, indicating that every $10 \%$ increase in the BCG index was associated with a $10.4 \%$ reduction in COVID-19 mortality.

Although intriguing, these findings must be interpreted with caution, as ecological studies carry inherent limitations. First, any risk reduction seen as a result of BCG vaccination applies to a population rather than an individual. Second, these studies are susceptible to bias from confounders such as differences in national demographics, reporting biases, testing rates for COVID-19 and the stage of the pandemic in each country. For example, an analysis of the COVID-19 spread rate in 74 countries stratified according to BCG vaccination policy (no policy, past universal vaccination policy or current universal policy $)^{149}$ reported that, although a lower COVID-19 spread rate and mortality was observed in countries with a current universal vaccination policy compared with countries with no or prior universal vaccination policy, adjusting for COVID-19 
testing rates meant that those countries with high testing rates (defined as 10 or more tests per thousand inhabitants) no longer showed a significant association between BCG policy and COVID-19 spread rate. Accordingly, in a study of Israeli adults aged 35-41 years with COVID-19-compatible symptoms, individuals born during a period of universal BCG vaccination had similar rates of positive COVID-19 test results to those who were born after cessation of universal vaccination $(11.7 \%$ versus $10.4 \%$, 95\% CI -0.3-2.9\%; $P=0.09)^{150}$. Likewise, a preliminary study was unable to demonstrate an association between COVID-19 case fatality and BCG vaccination before 1980 $(P=0.258)$ or with year of establishment of universal vaccination $(P=0.852)^{151}$. Several other studies have also failed to demonstrate a correlation between vaccination policy and SARS-CoV-2 infection ${ }^{152-154}$.

A 2021 study has attempted to reconcile the conflicting findings from different studies regarding protection of BCG against COVID-19 (REF. ${ }^{155}$ ). By modelling the effect of BCG vaccination across different times during the pandemic the authors show that the protective effect of $B C G$ vaccination against COVID-19 is strongest during the early stages of the pandemic. This protection gradually dissipates in later stages as governments introduce social distancing and other preventative measures.

Clinical data. Although results from ecological studies are conflicting, clinical data to support the hypothesis that BCG offers protection against COVID-19 are emerging. In a retrospective study, volunteers who received the BCG vaccine within the past 5 years had a lower incidence of self-reported sickness $(20.7 \%$ versus $31.1 \%, P<0.05)$ and extreme fatigue $(8.3 \%$ versus $18.9 \%, P<0.01)$ during the COVID-19 pandemic compared with those who had never received the vaccine ${ }^{156}$. Data from 120 consecutive adult patients with COVID-19 from a predominantly Latino and Hispanic population receiving care at a major federally qualified health centre demonstrated that BCG-vaccinated patients were less likely to require hospital admission during the disease course than non-BCG-vaccinated individuals ( $3.7 \%$ vs $15.8 \%, P=0.019$ ). This association remained unchanged after adjusting for demographics and comorbidities $(P=0.017)$ using multivariate regression analysis ${ }^{157}$. Accordingly, a study that assessed morbidity due to SARS-CoV-2 infections in a large cohort of health-care professionals from a multi-site Los Angeles health-care organization ${ }^{20}$ reported that almost $30 \%$ of the study participants had a history of BCG vaccination, and that BCG vaccination was associated with a decrease in self-reported COVID-19 diagnoses (1.9\% versus $2.9 \% ; P=0.029$ ), reduced seroprevalence of anti-SARS-CoV-2 IgG (2.7\% versus $3.8 \% ; P=0.044)$ and a decrease in COVID-19-specific symptoms $(75.6 \%$ versus $72.7 \%$; $P=0.017$ ).

Ultimately, these findings need confirmation and phase III-IV trials are currently underway to establish whether BCG vaccination of health-care workers and the elderly could protect them from COVID-19 (TABLE 1). If proved effective, $B C G$ vaccines could be repurposed to provide a rapid response to the pandemic ${ }^{158}$. However, the relevance of these trials has been called into question now that COVID-19-specific vaccinations are available. Published efficacy data from the BioNTech/Pfizer ${ }^{159}$ and Moderna ${ }^{160}$ trials indicate vaccine efficacy rates of approximately $95 \%$, with a third vaccine from The University of Oxford/AstraZeneca providing at least $62 \%$ protection $^{161}$. Following the launch of national COVID-19 vaccination programmes in several countries, the question of whether such BCG trials ought to continue should be considered. Several important arguments have been made in support of continuing these trials ${ }^{162}$. First, the duration of protection conferred by COVID-19 vaccines and the extent to which the vaccine response is affected by co-morbidities and frailty in the elderly is unknown. One possibility is that a combination of BCG with a COVID-19-specific vaccine might aid the immune response and induce lasting protection. Furthermore, global demand for COVID-19 vaccines is likely to vastly outstrip available supply, with the vaccine remaining inaccessible to almost one-quarter of the world's population until 2022 (REF. ${ }^{163}$ ). By contrast, BCG vaccines are widely available and might be useful to bridge the gap until production of COVID-19 vaccines is able to meet demand. Finally, establishing whether BCG vaccination is able to protect against COVID-19 severity might provide a tool for use against emerging pathogens in future pandemics.

\section{The outlook for BCG vaccination for} COVID-19. Until results from these trials are published, BCG vaccination should only be used for COVID-19 in a trial setting, according to the WHO guidance ${ }^{164}$; the BCG vaccine is already in short supply and use outside this setting risks jeopardizing supplies for TB prevention in endemic areas.
If trials demonstrate a positive treatment effect, a major concern for urologists f14land their patients will be how the supply of BCG for bladder cancer is affected. Urologists have faced global shortages of BCG for a decade and, in the short term, this shortage could be further exacerbated if BCG is repurposed for the COVID-19 effort. However, notably, the dose of BCG used for vaccination is $1 / 500$ th the dose used for bladder cancer treatment, meaning that, in theory, at least 500 subjects could be vaccinated from a single vial of $\mathrm{BCG}^{165}$. Furthermore, the renewed interest in BCG might encourage manufacturers who have hitherto been reluctant to increase production (or indeed, have left the market ${ }^{28}$ ) to become newly incentivized to increase BCG production, benefitting patients with bladder cancer and also increasing available vaccine supplies for $\mathrm{TB}$ prevention in endemic areas.

\section{Conclusions}

A century after the first use of BCG in humans, the vaccine has become established for the prevention of disseminated forms of TB and is also used intravesically as the standard treatment for high-risk NMIBC. Besides its use in TB and bladder cancer, the BCG vaccine might have beneficial non-specific effects and the mechanisms involved in such effects are now becoming better understood. Phase IV trials are currently underway to prospectively measure the role of these non-specific effects on protection against SARS-CoV-2 infection. Nevertheless, many questions regarding the mechanism of action of BCG remain, and it is hoped that these trials might provide new insights to improve our understanding of how to modulate the host response to prevent damage whilst protecting against infections, including COVID-19.

Niyati Lobo' ${ }^{1}$, Nathan A. Brooks' ${ }^{1}$, Alexandre R. Zlotta ${ }^{2,3}$ Jeffrey D. Cirillo ${ }^{4}$, Stephen Boorjian ${ }^{5}$, Peter C. Black ${ }^{6}$, Joshua J. Meeks', Trinity J. Bivalacqua ${ }^{8}$, Paolo Gontero ${ }^{9}$, Gary D. Steinberg ${ }^{10}$, David McConkey ${ }^{11}$, Marko Babjuk ${ }^{12}$, J. Alfred Witjes ${ }^{13}$ and Ashish M. Kamat (iD ${ }^{1 \otimes}$

'Department of Urology, MD Anderson Cancer Center, Houston, TX, USA.

${ }^{2}$ Division of Urology, Department of Surgery, Sinai Health System, University of Toronto, Toronto, ON, Canada

${ }^{3}$ Department of Surgical Oncology, Division of Urology, Princess Margaret Cancer Centre, University of Toronto and University Health Network, Toronto, ON, Canada.

${ }^{4}$ Department of Microbial Pathogenesis and Immunology, Texas A\&M Health Science Center, Bryan, TX, USA.

${ }^{5}$ Department of Urology, Mayo Clinic, Rochester, $M N, U S A$. 
${ }^{6}$ Vancouver Prostate Centre, Vancouver, BC, Canada. ${ }^{7}$ Northwestern University School of Medicine, Chicago, IL, USA.

${ }^{8}$ The James Buchanan Brady Urological Institute and Department of Urology, The Johns Hopkins School of Medicine, Baltimore, MD, USA.

${ }^{9}$ Division of Urology, Molinette Hospital, University of Torino School of Medicine, Torino, Italy.

${ }^{10}$ Department of Urology, NYU Langone Health, New York, NY, USA.

${ }^{11}$ Johns Hopkins Greenberg Bladder Cancer Institute, Brady Urological Institute, Johns Hopkins University, Baltimore, MD, USA.

${ }^{12}$ Department of Urology, Hospital Motol, Second Faculty of Medicine, Charles University, Prague, Czech Republic.

${ }^{13}$ Department of Urology, Radboud University Nijmegen Medical Centre, Nijmegen, Netherlands.

凶e-mail: akamat@mdanderson.org

https://doi.org/10.1038/s41585-021-00481-1

Published online 15 June 2021

1. World Health Organization. BCG vaccine. $W H O$ https://www.who.int/biologicals/areas/vaccines/bcg/en/ (2018).

2. Trunz, B. B., Fine, P. \& Dye, C. Effect of BCC vaccination on childhood tuberculous meningitis and military tuberculosis worldwide: a meta-analysis and assessment of cost-effectiveness. Lancet 367 1173-1180 (2006)

3. Babjuk, M. et al. EAU Guidelines on non-muscle invasive bladder cancer (TaT1 and carcinoma in situ) - 2019 update. Eur. Urol. 75, 639-657 (2019).

4. World Health Organization. Coronavirus disease (COVID-19) pandemic. WHO https://www.who.int/ emergencies/diseases/novel-coronavirus-2019 (2020).

5. Dong, E., Du, H. \& Gardner, L. An interactive webbased dashboard to trace COVID-19 in real time. Lancet 20, 533-534 (2020).

6. van't Wout, J., Poell, R. \& Furth, R. The role of BCG PPD-activated macrophages in resistance against systemic candidiasis in mice. Scand. J. Immunol. 36 713-720 (1992).

7. Kleinnijenhuis, J., Quintin, J. \& Preijers, F. Bacille Calmette-Guerin induces NOD2-dependent nonspecific protection from reinfection via epigenetic reprogramming of monocytes. Proc. Natl Acad. Sci. USA 109, 17537-17542 (2012).

8. Tribouley, J., Tribouley-Duret, J. \& Appriou, M. Effect of Bacillus Calmette Guerin (BCG) on the receptivity of nude mice to Schistosoma mansoni. C. R. Seances Soc. Biol. Fil. 172, 902-904 (1978).

9. Clark, I. A., Allison, A. C. \& Cox, F. E. Protection of mice against Babesia and Plasmodium with BCG. Nature 259, 309-311 (1976).

10. Matsumoto, S. et al. Mycobacterium bovis Bacillus Calmette-Guerin induces protective immunity against infection by Plasmodium yoelii at blood-stage depending on shifting immunity toward Th 1 type and inducing protective IgG2a after the parasite infection. Vaccine 19, 779-787 (2000).

11. Parra, M. et al. Molecular analysis of non-specific protection against murine malaria induced by BCG vaccination. PLOS ONE 8, e66115 (2013).

12. Walk, J. et al. Outcomes of controlled human malaria infection after BCG vaccination. Nat. Commun. 10, 874 (2019).

13. Leentjens, J. et al. BCG vaccination enhances the immunogenicity of subsequent influenza vaccination in healthy volunteers: a randomized, placebo-controlled pilot study. J. Infect. Dis. 212, 1930-1938 (2015).

14. Arts, R. J. W. et al. BCG vaccination protects against experimental viral infection in humans through the induction of cytokines associated with trained immunity. Cell Host Microbe 23, 89-100.e5 (2018).

15. Giamarellos-Bourboulis, E. J. et al. Activate: randomized clinical trial of BCG vaccination against infection in the elderly. Cell. 183, 315-323 (2020).

16. Hegarty P et al COVID-19 and Bacillus CalmetteGuerin: what is the link? Eur Urol Oncol. 3, 259-261 (2020).

17. Berg, M. et al. Mandated Bacillus Calmette-Guérin (BCG) vaccination predicts flattened curves for the spread of COVID-19. Sci. Adv. 6, eabc1463 (2020).
18. Ozdemir, C., Kucuksezer, U. C. \& Tamay, Z. U. Is $B C G$ vaccination affecting the spread and severity of COVID-19? Allergy 75, 1824-1827 (2020).

19. Escobar, L. E., Molina-Cruz, A. \& Barillas-Mury, C BCG vaccine protection from severe coronavirus disease 2019 (COVID-19). Proc. Natl Acad. Sci. USA 117, 17720-17726 (2020).

20. Rivas, M. N. et al. BCG vaccination history associates with decreased SARSCoV-2 seroprevalence across a diverse cohort of healthcare workers. J. Clin. Invest. 131, e145157 (2021).

21. Calmette, A., Bocquet, A. \& Negre, L. Contribution a l'etude du bacilli tuberculuex bilie. Ann. Inst. Pasteur 9, 651-670 (1921)

22. Lange, B. Nouvelles recherche sur les causes des accidents de Lubeck. Rev. Tuberc. Extrait 12, 1142-1170 (1931)

23. Fine, P. E. M., Carneiro, I. A. M., Milstien, J. B. $\&$ Clements, C. J. Issues relating to the use of BCG in immunization programmes. 1-44 (World Health Organization, 1999).

24. Plotkin, S. A., Orenstein, W. A. \& Offit, P. A. Vaccines. 6th Edn. (Elsevier Saunders, 2013).

25. Brosch, R. B. et al. Genome plasticity of BCG and impact on vaccine efficacy. PNAS. 104, 5596-560 (2007).

26. Ritz, N. \& Curtis, N. Mapping the global use of different BCG vaccine strains. Tuberculosis $\mathbf{8 9}$, 248-251 (2009).

27. World Health Organization. Information Sheet. Observed rate of vaccine reactions. Bacille CalmetteGuerin (BCG) vaccine. WHO https://www.who.int/ vaccine_safety/initiative/tools/BCG_Vaccine_rates information_sheet.pdf (2012).

28. Messing, E. M. The BCG shortage. Bladder Cancer 3, 227-228 (2017)

29. Roth, A. et al. Vaccination technique, PPD reaction and BCG scarring in a cohort of children born in Guinea-Bissau 2000-2002. Vaccine 23, 3991-3998 (2005).

30. Anderson, $\mathrm{E}$. J. et al. The influence of BCG vaccine strain on mycobacteria-specific and non-specific immune responses in a prospective cohort of infants in Uganda. Vaccine 30, 2083-2089 (2012).

31. Frankel, H. et al. Different effects of BCG strains - a natural experiment evaluating the impact of the Danish and the Russian BCG strains on morbidity and scar formation in Guinea-Bissau. Vaccine 34 , 4586-4593 (2016).

32. Shann, F. Editorial commentary: different strains of bacillus calmette-guerin vaccine have very different effects on tuberculosis and on unrelated infections. Clin. Infect. Dis. 61, 960-962 (2015).

33. Funch, K. M. et al. Determinants of BCG scarification among children in rural Guinea-Bissau: a prospective cohort study. Hum. Vaccin. Immunother. 14 , 2434-2442 (2018)

34. Schaltz-Buccholzer, F. et al. Early vaccination with bacille calmette-guerin-denmark or BCG-Japan versus BCG-Russia to healthy newborns in Guinea-Bissau: randomized controlled trial. Clin. Infec. Dis. $\mathbf{7 1}$ 1883-1893 (2020).

35. Comstock, G. W. Simple, practical ways to assess the protective efficacy of a new tuberculosis vaccine. Clin. Infect. Dis. 30, S250-S253 (2000)

36. Favorov, M. et al. Comparative tuberculosis (TB) prevention effectiveness in children of Bacillus Calmette-Guérin (BCG) vaccines from different sources, Kazakhstan. PLoS ONE 7, e32567 (2012).

37. Gan, C. et al. BCG immunotherapy for bladder cancer - the effects of substrain differences. Nat. Rev. Urol. 10, 580-588 (2013)

38. Rentsch, C. A. et al. Bacillus Calmette-Guérin strain differences have an impact on clinical outcome in bladder cancer immunotherapy. Eur. Urol. 66, 677-688 (2014)

39. Witjes, J. A. et al. The efficacy of BCG TICE and BCC Connaught in a cohort of 2,099 patients with T1G3 non-muscle-invasive bladder cancer. Urol. Oncol. 34 , 484 (2016).

40. D'Andrea, D. et al. Comparative effectiveness of intravesical BCG-Tice and BCG-Moreau in patients with non-muscle-invasive bladder cancer. Clin. Genitourin. Cancer 18, 20-25 (2020).

41. Boehm, B. E. et al. Efficacy of bacillus Calmette-Guerin strains for treatment of nonmuscle invasive bladder cancer: a systematic review and network meta-analysis. J. Urol. 198, 503-510 (2017)

42. World Health Organisation. Tuberculosis. WHO https://www.who.int/news-room/fact-sheets/detail/ tuberculosis (2021)
43. Mangtani, P. et al. Protection by BCG vaccine against tuberculosis: a systematic review of randomized controlled trials. Clin. Infect. Dis. 58, 470-480 (2014).

44. Abubakar, I. et al. Systematic review and metaanalysis of the current evidence on the duration of protection by bacillus Calmette-Guerin vaccination against tuberculosis. Health Technol. Assess. 17 1-372 (2013)

45. Aronson, N. E. et al. Long-term efficacy of BCG vaccine in American Indians and Alaska Natives: a 60-year follow-up study. JAMA 291, 2086-2091 (2004).

46. Nguipdop-Djomo, P., Heldal, E., Rodrigues, L. C., Abubakar, I. \& Mangtani, P. Duration of BCG protection against tuberculosis and change in effectiveness with time since vaccination in Norway: a retrospective population-based cohort study. Lancet Infect. Dis. 16, 219-226 (2016).

47. Swaminathan, S. \& Rekha, B. Pediatric tuberculosis: global overview and challenges. Clin. Infect. Dis. 50, S184-S194 (2010).

48. Fine, P. E. Variation in protection by BCG: implications of and for heterologous immunity. Lancet 346 , 1339-1345 (1995).

49. Brandt, L. et al. Failure of the Mycobacterium bovis BCG vaccine: some species of environmental mycobacteria block multiplication of BCG and induction of protective immunity to tuberculosis. Infect. Immun. 70, 672-678 (2002)

50. Weir, R. E. et al. The influence of previous exposure to environmental mycobacteria on the interferon-gamma response to bacille Calmette-Guérin vaccination in southern England and northern Malawi. Clin. Exp. Immunol. 146, 390-399 (2006).

51. Nemes, E. et al. Prevention of M. Tuberculosis infection with $\mathrm{H} 4$ :IC31 vaccine or BCG revaccination. N. Engl. J. Med. 379, 138-149 (2018).

52. Van Der Meeren, O. et al. Phase $2 \mathrm{~b}$ controlled trial of $\mathrm{M72} / \mathrm{ASO}_{\mathrm{E}}$ vaccine to prevent tuberculosis. N. Engl. J. Med. 379, 1621-1634 (2018)

53. Darrah, P. A. et al. Prevention of tuberculosis in macaques after intravenous BCG immunization. Nature 577, 95-102 (2020).

54. Benn, C. S. et al. A small jab - a big effect: nonspecific immunomodulation by vaccines. Trends Immunol. 34, 431-439 (2013).

55. Naslund, C. Resultats des experiences de vaccination par le BCG poursuivies dans le Norrbotten (Suède) (Septembre 1927-Décembre 1931). Vaccination Preventative de Tuberculose, Rapports et Documents (Institut Pasteur, 1932)

56. Hirve, S. et al. Non-specific and sex-differential effects of vaccinations on child survival in rural western India. Vaccine. 30, 7300-7308 (2012).

57. Moulton, L. H. et al. Evaluation of non-specific effects of infant immunizations on early infant mortality in a southern Indian population. Trop. Med. Int. Health 10 947-955 (2005)

58. Kristensen, I., Aaby, P. \& Jensen, H. Routine vaccinations and child survival: follow up study in Guinea-Bissau, West Africa. BMJ 321, 1435-1438 (2000).

59. de Castro, M. J., Pardo-Seco, J \& Martinon-Torrest, F. Nonspecific (heterologous) protection of neonatal BCC vaccination against hospitalization due to respiratory infection and sepsis. Clin. Infect. Dis. 60, 1611-1619 (2015).

60. Roth, A. et al. Low birth weight infants and Calmette Guerin bacillus vaccination at birth: community study from Guinea-Bissau. Pediatr. Infect. Dis. J. 23 544-550 (2004).

61. Aaby, P. et al. Randomized trial of BCG vaccination at birth to low-birth-weight children: Beneficial nonspecific effects in the neonatal period? J. Infect Dis. 2, 245-252 (2011)

62. Biering-Sørensen, S. et al. Small randomized trial among low-birth-weight children receiving bacillus Calmette-Guérin vaccination at first health center contact. Pediatr. Infect. Dis. J. 31, 306-308 (2012).

63. Jensen, K. J. et al. Heterologous immunological effects of early BCG vaccination in low-birth-weight infants in Guinea-Bissau: a randomized-controlled trial. J. Infect. Dis. 211, 956-967 (2014).

64. Netea, M. G., van der Meer, J. W., van Deuren, M. \& Kullberg, B. J. Proinflammatory cytokines and sepsis syndrome: not enough, or too much of a good thing? Trends Immunol. 24, 254-258 (2003).

65. van der Poll, T., van de Veerdonk, F. L., Scicluna, B. P. $\&$ Netea, M. G. The immunopathology of sepsis and potential therapeutic targets. Nat. Rev. Immunol. 17 407-420 (2017) 
66. Van Puffelen, J. H. et al. Trained immunity as a molecular mechanism for BCG immunotherapy in bladder cancer. Nat. Rev. Urol. 17, 513-525 (2020).

67. Aaby, P. et al. Early BCG vaccination and reduction in atopy in Guinea-Bissau. Clin. Exp. Allergy 30 644-650 (2000)

68. Steenhuis, T. J. et al. Bacille-Calmette-Guerin vaccination and the development of allergic disease in children: a randomized, prospective, single-blind study. Clin. Exp. Allergy 38, 79-85 (2008)

69. Thøstesen, L. M. et al. Neonatal BCG vaccination and atopic dermatitis before 13 months of age: a randomized clinical trial. Allergy 73, 498-504 (2018).

70. Usher, N. T. et al. Association of BCG vaccination in childhood with subsequent cancer diagnoses: a 60 year follow-up of a clinical trial. JAMA Netw. Open 2, e1912014 (2019).

71. Coley, W. B. The treatment of malignant tumors by repeated inoculations of erysipelas. With a report of ten original cases. 1893. Clin. Orthop. Relat. Res. 262, 3-11 (1991).

72. Coley, W. B. The treatment of inoperable sarcoma by bacterial toxins (the mixed toxins of the Streptococcus erysipelas and the Bacillus prodigiosus). Proc. $R$. Soc. Med. 3, 1-48 (1910).

73. Pearl, R. Cancer and tuberculosis. Am. J. Hygiene $\mathbf{9}$ 97 (1929).

74. Old, L. J., Clarke, D. A. \& Benacerraf, B. Effect of bacillus Calmette-Guerin infection on transplanted tumours in the mouse. Nature 184, 291-292 (1959).

75. Zbar, B. \& Tanaka, T. Immunotherapy of cancer: regression of tumors after intralesional injection of living Mycobacterium bovis. Science 172, 271-273 (1971).

76. Zbar, B. \& Rapp, H. J. Immunotherapy of guinea pig cancer with BCG. Cancer 34, 1532-1540 (1974).

77. Mathe, G. et al. Active immunotherapy for acute lymphoblastic leukemia. Lancet 1, 697-699 (1969).

78. Morton, D. L. BCG immunotherapy of malignant melanoma: summary of a seven-year experience. Ann. Surg. 180, 635-641 (1974).

79. Galligioni, E. et al. Adjuvant immunotherapy treatment of renal carcinoma patients with autologous tumor cells and bacillus Calmette-Guerin: five year results of a prospective randomized study. Cancer 12 , 2560-2566 (1996).

80. Gray, B. N. et al. Controlled clinical trial of adjuvant immunotherapy with BCG and neuraminidase-treated autologous tumour cells in large bowel cancer. J. Surg Oncol. 40, 34-37 (1989).

81. Maurer, L. H. et al. Combined modality therapy with radiotherapy, chemotherapy, and immunotherapy in limited small-cell carcinoma of the lung: a Phase III Cancer and Leukemia Group B Study. J. Clin. Oncol. 7 969-976 (1985)

82. Gandhi, N. M., Morales, A. \& Lamm, D. L. Bacillus Calmette-Guerin immunotherapy for genitourinary cancer. BJU Int. 112, 288-297 (2013).

83. Coe, J. E. \& Feldman, J. D. Extracutaneous delayed hypersensitivity, particularly in the guinea-pig bladder. Immunology 10, 127-136 (1966).

84. Dekernion, J. B. et al. Successful transurethral intralesional BCG therapy of a bladder melanoma. Cancer 36, 1662-1667 (1975).

85. Morales, A., Eidinger, D. \& Bruce, A. W. Intracavitary Bacillus Calmette-Guerin in the treatment of superficial bladder tumors. J. Urol. 116, 180-183 (1976).

86. Lamm, D. L. et al. Bacillus Calmette-Guerin immunotherapy of superficial bladder cancer. J. Urol. 124, 38-40 (1980).

87. Pinsky, C. M. et al. Intravesical administration of bacillus Calmette-Guerin in patients with recurrent superficial carcinoma of the urinary bladder: report of a prospective, randomized trial. Cancer. Treat. Rep. 69, 47-53 (1985).

88. Shelley, M. D. et al. A systematic review of intravesical bacillus Calmette-Guerin plus transurethral resection vs transurethral resection alone in Ta and $\mathrm{T} 1$ bladder cancer. BJU Int. 88, 209 (2001).

89. Han, R. F. et al. Can intravesical bacillus CalmetteGuerin reduce recurrence in patients with superficial bladder cancer? A meta-analysis of randomized trials. Urology 67, 1216-1223 (2006)

90. Bohle, A. et al. Intravesical bacillus Calmette-Guerin versus mitomycin $C$ for superficial bladder cancer a formal meta-analysis of comparative studies on recurrence and toxicity. J. Urol. 169, 90-95 (2003).

91. Shelley, M. D. et al. Intravesical bacillus CalmetteGuerin is superior to mitomycin $C$ in reducing tumour recurrence in high-risk superficial bladder cancer: a meta-analysis of randomized trials. BJU Int. 93 485-490 (2004).

92. Malmstrom, P. U. et al. An individual patient data meta-analysis of the long-term outcome of randomised studies comparing intravesical mitomycin $C$ versus bacillus Calmette-Guerin for non-muscle-invasive bladder cancer. Eur. Urol. 56, 247-256 (2009).

93. Sylvester, R. J. et al. Intravesical bacillus CalmetteGuerin reduces the risk of progression in patients with superficial bladder cancer: a meta-analysis of the published results of randomized clinical trials. J. Urol. 168, 1964-1970 (2002).

94. Bohle, A. et al. Intravesical bacille Calmette-Guerin versus mitomycin $\mathrm{C}$ in superficial bladder cancer: formal meta-analysis of comparative studies on tumor progression. Urology 63, 682-686 (2004).

95. Ehdaie, B., Sylvester, R. \& Herr, H. W. Maintenance Bacillus Calmette-Guerin treatment of non-muscle invasive bladder cancer: a critical evaluation of the evidence. Eur. Urol. 64, 579-585 (2013).

96. Lamm, D. L. et al. Maintenance BCG immunotherapy in recurrent $\mathrm{Ta}, \mathrm{T} 1$ and carcinoma in situ transitional cell carcinoma: a randomized Southwest Oncology Group Study. J. Urol. 163, 1124-1129 (2000).

97. Oddens, J. et al. Final results of an EORTC-GU cancers group randomized study of maintenance bacillus Calmette-Guerin in intermediate- and high-risk Ta, T1 papillary carcinoma of the urinary bladder: one third dose versus full dose and 1 year versus 3 years of maintenance. Eur. Urol. 63, 462-472 (2013).

98. Chang, S. S. et al. Diagnosis and treatment of nonmuscle invasive bladder cancer: AUA/SUO guideline. J. Urol. 196, 1021-1029 (2016)

99. Lamm, D. L. et al. A randomized trial of intravesical doxorubicin and immunotherapy with bacille Calmette-Guerin for transitional-cell carcinoma of the bladder. N. Engl. J. Med. 325, 1205-1209 (1991).

100. Lamm, D. L. Long-term results of intravesical therapy for superficial bladder cancer. Urol. Clin. North Am. 19, 573-580 (1992)

101. Cambier, S. et al. EORTC nomograms and risk groups for predicting recurrence, progression and diseasespecific and overall survival in non-muscle-invasive stage Ta-T1 urothelial bladder cancer patients treated with 1-3 years of maintenance Bacillus CalmetteGuerin. Eur. Urol. 69, 60-69 (2016).

102. Van der Meijden, A. P. et al. Maintenance Bacillus Calmette-Guerin for Ta T1 bladder tumors is not associated with increased toxicity: results from a European Organisation for Research and Treatment of Cancer Genito-Urinary Group Phase III Trial. Eur. Urol. 44, 429-434 (2003).

103. Brausi, M. et al. Side Effects of Bacillus CalmetteGuérin (BCG) in the Treatment of Intermediate- and High-risk Ta, T1 papillary carcinoma of the bladder: results of the EORTC genito-urinary cancers group randomised phase 3 study comparing one-third dose with full dose and 1 year with 3 years of maintenance BCG. Eur. Urol. 65, 69-76 (2014).

104. Gonzalez, O. Y. et al. Spectrum of Bacille CalmetteGuerin (BCG) infection after intravesical BCG immunotherapy. Clin. Infect. Dis. 36, 140-148 (2003).

105. Marquez-Batalla, S., Fraile-Villarejo, E., Belhassen-Garcia, M., Gutierrez-Zubiaurre, N. $\&$ Cordero-Sanchez, M. Disseminated infection due to Mycobacterium bovis after intravesical BCC instillation. World J. Clin Cases 2, 301-303 (2014).

106. Lamm, D. L. et al. Incidence and treatment of complications of Bacillus Calmette-Guerin intravesical therapy in superficial bladder cancer. J. Urol. 147, 596-600 (1992).

107. Kavoussi, L. R., Brown, E. J., Ritchey, J. K. $\delta$ Ratliff, T. L. Fibronectin-mediated Calmette-Guerin bacillus attachment to murine bladder mucosa. Requirement for the expression of an antitumo response. J. Clin. Invest. 85, 62-67 (1990).

108. Witjes, J. A. et al. Influence of fibrin clot inhibitors on the efficacy of intravesical Bacillus Calmette-Guerin in the treatment of superficial bladder cancer. The Dutch Southeast Cooperative Urological Group. Eur. Urol. 23, 366-370 (1993)

109. Lipsky, M. J. et al. The effect of fibrin clot inhibitors on the immunomodulatory efficacy of Bacillus CalmetteGuerin therapy for non-muscle invasive bladder cancer. Urology 81, 1273-1278 (2013).

110. Pettenati, C. \& Ingersoll, M. A. Mechanisms of BCG immunotherapy and its outlook for bladder cancer. Nat. Rev. Urol. 15, 615-625 (2018).

111. El-Demiry, M. I. et al. Local immune responses after intravesical BCG treatement for carcinoma in situ. Br. J. Urol. 60, 543-548 (1987).
112. Prescott, S. et al. HLA-DR expression by high grade superficial bladder cancer treated with BCG. Br. J. Urol. 63, 264-2269 (1989).

113. Jackson, A. M. et al. Induction of ICAM 1 expression on bladder tumours by BCG immunotherapy. J. Clin. Pathol. 47, 309-312 (1994).

114. Kamat, A. M. et al. Cytokine panel for response to intravesical therapy (CyPRIT): nomogram of changes in urinary cytokine levels predicts patient response to Bacillus Calmette-Guerin. Eur. Urol. 69, 197-200 (2016).

115. Redelman-Sidi, G., Glickman, M. S. \& Bochner, B. H. The mechanism of action of BCG therapy for bladder cancer- a current perspective. Nat. Rev. Urol. 11, 153-162 (2014).

116. Netea, M. G., Quintin, J. \& Van Der Meer, J. W. M. Trained immunity: a memory for innate host defense. Cell Host Microbe 9, 355-361 (2011).

117. Kleinnijenhuis, J., Quintin, J. \& Preijers, F. BCGinduced trained immunity in NK cells: role for nonspecific protection to infection. Clin. Immunol. 155, 213-219 (2014)

118. Netea, M. G. et al. Trained immunity: a program of innate immune memory in health and disease. Science 352, aaf1098 (2016).

119. Arts, R. J. W. et al. Immunometabolic pathways in BCG-induced trained immunity. Cell Rep. 17, 2562-2571 (2016).

120. Kaufmann, E. et al. BCG educates haematopoietic stem cells to generate protective innate immunity against tuberculosis. Cell 172, 176-190.

121. Cirovic, B. et al. BCG vaccination in humans elicits trained immunity vi the haematopoietic progenitor compartment. Cell Host Microbe. 28, 322-334 (2020).

122. Cheng, S. et al. mTOR/HIF1 a-mediated aerobic glycolysis as metabolic basis for trained immunity. Science 345, 1250684 (2014).

123. Li, T. \& Chen, Z. J. The cGAS-cGAMP-STING pathway connects DNA damage to inflammation, senescence, and cancer. J. Exp. Med. 215 , 1287-1299 (2018).

124. Paludan, S. R. \& Bowie, A. J. Immune sensing of DNA. Immunity 38, 70-80 (2013)

125. Ishikawa, H. \& Barber, G. N. STING is an endoplasmic reticulum adaptor that facilitates innate immune signalling. Nature 455, 674-678 (2008).

126. Ishikawa, H., Ma, Z. \& Barber, G. N. STING regulates intracellular DNA-mediated, type I interferondependent innate immunity. Nature 461, 788-792 (2009).

127. Li, X. D. et al. Pivotal roles of cCAS-cGAMP signaling in antiviral defense and immune adjuvant effects. Science 341, 1390-1394 (2013).

128. Dey, R. J. et al. Bacillus Calmette-Guerin overexpressing an endogenous stimulator of interferon genes agonist provides enhanced protection against pulmonary tuberculosis. J. Infect. Dis. 221 1048-1056 (2020).

129. Gröschel, M. I. et al. Recombinant BCG expressing ESX-1 of Mycobacterium marinum combines low virulence with cytosolic immune signaling and improved TB protection. Cell Rep. 18, 2752-2765 (2017).

130. Loxton, A. G. et al. Safety and immunogenicity of the recombinant Mycobacterium bovis BCG vaccine VPM1002 in HIV-unexposed newborn infants in South Africa. Clin. Vaccine Immunol. 24, e00439-16 (2017).

131. US National Library of Medicine. ClinicalTrials.gov https://clinicaltrials.gov/ct2/show/NCT04387409 (2020)

132. US National Library of Medicine. ClinicalTrials.gov https://clinicaltrials.gov/ct2/show/NCT04439045 (2021)

133. US National Library of Medicine. ClinicalTrials.gov https://clinicaltrials.gov/ct2/show/NCT04435379 (2021)

134. Conti, P. et al. Bacillus Calmette-Guerin potentiates monocyte responses to lipopolysaccharide- induced tumor necrosis factor and interleukin-1, but not interleukin- 6 in bladder cancer patients. Cancer Immunol. Immunother. 38, 365-371 (1994).

135. Kim, C. I., Shin, J. S., Kim, H. I., Lee, J. M. \& Kim, S. J. Production of tumor necrosis factor by intravesical administration of bacillus Calmette-Guerin in patients with superficial bladder cancer. Yonsei Med. J. 34, 356-364 (1993).

136. Calais da Silva, F. M. et al. Systemic humoral responses of non-muscle-invasive bladder cancer during BCG treatment: less is more. J. Cancer Metastasis Treat. 3, 116-126 (2017). 
137. Reale, M. et al. Production of MCP-1 and RANTES in bladder cancer patients after bacillus CalmetteGuerin immunotherapy. Cancer Immunol. Immunother 51, 91-98 (2002).

138. de Reijke, T. M. et al. Urinary cytokines during intravesical bacillus Calmette-Guerin therapy for superficial bladder cancer: processing, stability and prognostic value. J. Urol. 155, 477-482 (1996).

139. Koti, M. et al. Investigating the STING pathway to explain mechanisms of BCG failures in non-muscle invasive bladder cancer: prognostic and therapeutic implications. Bladder Cancer 5, 225-234 (2019).

140. Singh, A. K., Praharaj, M. \& Lombardo, K. A Recombinant BCG overexpressing a STING agonist elicits trained immunity and improved anti-tumour efficacy in non-muscle invasive bladder cancer. Urol. Oncol. 38, 899 (2020).

141. McKibbin, W. J. \& Fernando, R. The globa macroeconomic impacts of COVID-19: seven scenarios. Preprint at SSRN https://doi.org/10.2139 ssrn.3547729 (2020)

142. Gilbert, M. et al. Preparedness and vulnerability of African countries against importations of COVID-19. a modelling study. Lancet 395, 871-877 (2020).

143. Moore, K. A., Lipsitch, M., Barry, J. M. \& Osterholm, M. T. COVID-19: the CIDRAP Viewpoint. https:/www.cidrap umn edu/sites/default/files/public/ downloads/cidrap-covid19-viewpoint-part1_0.pdf (2020).

144. Wu, C. et al. Risk factors associated with acute respiratory distress syndrome and death in patients with coronavirus disease 2019 pneumonia in Wuhan, China. JAMA Intern. Med. 180, 934-943 (2020)

145. Kamat, A. M. et al. Bladder cancer. Lancet 388 2796-2810 (2016)

146. Ribal, M. J. et al. EAU Guidelines Office Rapid Reaction Group: An organization wide collaborative effort to adapt the EAU guidelines recommendations to the COVID-19 era. https://uroweb.org/wp-content uploads/EAU-Guidelines-Office-Rapid-ReactionGroup-An-organisation-wide-collaborative-effortto-adapt-the-EAU-guidelines-recommendations-to-theCOVID-19-era.pdf (2020).

147. Lenfant, L. et al. Adjustments in the use of intravesical instillations of Bacillus Calmette-Guerin for high-risk non-muscle-invasive bladder cancer during the COVID-19 pandemic. Eur. Urol. 78, 1-3 (2020)

148. Wallis, C. J. D. et al. Risks from deferring treatment for genitourinary cancers: a collaborative review to aid trige and management during the COVID-19 pandemic. Eur. Urol. 78, 29-42 (2020).

149. Hensel, J. et al. Protection against SARS-CoV-2 by BCG vaccination is not supported by epidemiological analyses. Sci. Rep. 10, 18377 (2020)

150. Hamiel, U., Kozer, E. \& Youngster, I. SARS-CoV-2 rates in BCG-vaccinated and unvaccinated young adults. JAMA 323, 2340-2341 (2020).

151. Szigeti, R., Kellermayer, D., Trakimas, G. $\delta$ Kellermayer, R. BCG epidemiology supports its protection against COVID-19? A word of caution. PLOS ONE 15, e0240203 (2020).

152. Wassenaar, T. M., Buzard, G. S. \& Newman, D. J. BCC vaccination early in life does not improve COVID-19 outcome of elderly populations, based on nationally reported data. Lett. Appl. Microbiol. 71, 498-505 (2020).

153. Lindestam Arlehamn, C. S., Sette, A \& Peters, B. Lack of evidence for $B C G$ vaccine protection from severe COVID-19. Proc. Natl Acad. Sci. USA 117 25203-25204 (2020).

154. De Chaisemartin, C. \& de Chaisemartin, L. BCG vaccination in infancy does not protect against COVID-19. Evidence from a natural experiment in Sweden. Clin. Infect. Dis. https://doi.org/10.1093/ $\mathrm{cid} / \mathrm{ciaa} 1223$ (2020).

155. Fu, W. et al. Reconcile the debate over protective effects of BCG against COVID-19. Sci. Rep. 11, 8356 (2021)

156. Moorlag, S. et al. Safety and COVID-19 symptoms in individuals recently vaccinated with BCC: a retrospective cohort study. Cell Rep. Med. 1, 100073 (2020).

157. Weng, C. \& Chan, P. A. BCG as an adjunct or alternative vaccine to prevent COVID-19? J. Travel Med. 27, taaa 175 (2021)

158. Curtis, N. et al. Considering BCG vaccination to reduce the impact of COVID-19. Lancet 395, 1545-1546 (2020).

159. Polack, F. P. et al. Safety and efficacy of the BNT162b2 mRNA Covid-19 vaccine. N. Engl. J. Med. 383, 2603-2615 (2020).

160. Baden, L. R. et al. Efficacy and safety of the mRNA1273 SARS-CoV-2 vaccine. N. Engl. J. Med. 384, 403-416 (2021)

161. Voysey, M. et al. Safety and efficacy of the ChAdOx $n C o V-19$ vaccine (AZD 1222) against SARS-CoV2: an interim analysis of four randomized controlled trials in Brazil, South Africa and the UK. Lancet 397, 99-111 (2021).

162. Netea, M. G., van der Meer, J. W. M. \& van Crevel, R. $B C G$ vaccination in healthcare providers and the protection against COVID-19. J. Clin. Invest. 131 e145545 (2021)

163. So, A. D. \& Woo, J. Reserving coronavirus disease 2019 vaccines for global access: cross sectional analysis. BMJ. 371, m4750 (2020).

164. World Health Organization. Bacille Calmette-Guerin (BCG) vaccination and COVID-19. WHO https://www who.int/news-room/commentaries/detail/bacillecalmette-gu\%C3\%A9rin-(bcg)-vaccination-andcovid-19 (2020).

165. Brooks, N. A. et al. The role of the urologist, BCC vaccine administration and SARS-CoV-2: an overview. BJUI Compass 1, 87-92 (2020).

166. US National Library of Medicine. ClinicalTrials.gov https://clinicaltrials. gov/ct2/show/NCT0 4350931 (2020)

167. US National Library of Medicine. ClinicalTrials.gov https://clinicaltrials.gov/ct2/show/NCT04384549 (2020)

168. US National Library of Medicine. ClinicalTrials.gov https://clinicaltrials.gov/ct2/show/NCT0 4328441 (2021)

169. US National Library of Medicine. ClinicalTrials.gov https://clinicaltrials.gov/ct2/show/NCT04379336 (2020)

170. US National Library of Medicine. ClinicalTrials.gov https://clinicaltrials.gov/ct2/show/NCT04537663 (2021)

171. US National Library of Medicine. ClinicalTrials.gov https://clinicaltrials.gov/ct2/show/NCT04648800 (2020)

172. US National Library of Medicine. ClinicalTrials.gov https://clinicaltrials.gov/ct2/show/NCT04414267 (2021)

173. US National Library of Medicine. ClinicalTrials.gov https://clinicaltrials. gov/ct2/show/NCT0 4417335 (2020)

174. US National Library of Medicine. ClinicalTrials.gov https://clinicaltrials.gov/ct2/show/NCT04373291 (2021)

175. US National Library of Medicine. ClinicalTrials.gov https://clinicaltrials.gov/ct2/show/NCT04641858 (2020)

176. US National Library of Medicine. ClinicalTrials.gov https://clinicaltrials. gov/ct2/show/NCT04542330 (2020)
177. US National Library of Medicine. ClinicalTrials.gov https:/clinicaltrials oov/ct2/show/NCT04659941 (2020)

178. US National Library of Medicine. ClinicalTrials.gov https://clinicaltrials.gov/ct2/show/NCT04534803 (2021)

179. US National Library of Medicine. ClinicalTrials.gov https://clinicaltrials.gov/ct2/show/NCT04369794 (2020)

180. O’Neill, L. A. J. \& Netea, M. G. BCG-induced trained immunity: can it offer protection against COVID-19? Nat. Rev. Immunol. 20, 335-337 (2020).

\section{Author contributions}

N.L., and N.A.B. researched data for article; N.L., N.A.B. and A.M.K. wrote the article; all authors made substantial contributions to discussion of the content and reviewed and edited the manuscript before submission.

\section{Competing interests}

S.B. declares that he has acted as a consultant for Ferring Sanofi, ArTara and FerCene. P.C.B. declares that he is an advisory board member or equivalent for AbbVie, Asieris, AstraZeneca, Astellas, Bayer, Biosyent, BMS, EMD-Serono, Ferring, Fergene, H3-Biomedicine, Janssen, Merck, Roche, Sanofi, Urogen. He has received grant support or honoraria from iProgen, Sanofi, Bayer and CSK. P.C.B also holds a patent with Decipher Biosciences. J.J.M. declares that he acts as a consultant for Merck, AstraZeneca, Ferring, Cold Genesys, Janssen, Nucleix and Foundation Medicine. He has received research funding from AbbVie, Tesaro and Epizyme. D.M. declares that he has received grant support from AstraZeneca, Rainer Pharmaceuticals and honoraria from Janssen, Rainier Pharmaceuticals and H3 biomedicine. T.J.B. declares that he has acted as a consultant for FerGene, Genesis Biotechnology and Photocure. G.D.S declares that he is a member of clinical trial protocol committees for Merck, BMS, Janssen, Cold Genesys, Pfizer, PhotoCure and Fidia. He is or has been a scientific advisor/consultant within the past 5 years for Heat Biologics, Cold Genesys, PhotoCure, Merck, Roche/Genentech, Ciclomed, Taris Biomedical, MDxHealth, Fidia Farmaceuticals, Urogen, Ferring, Aduro, Boston Scientific, Bristol Myers Squibb, AstraZeneca, Pfizer, Janssen, Epivax Oncology, Natera, FKD, Ferring, EnGene Bio, SesenBio, BioCanCell, Nucleix, Ipsen, Combat Medical, Astellas, Fergene, Dendreon, AbbVie and Seattle Genetics. A.M.K. is a consultant or advisory board member for Abbott Molecular, Arquer Diagnostics, ArTara Therapeutics, Asieris Pharmaceuticals, AstraZeneca, BioClin Therapeutics, Bristol Myers Squibb, Cepheid, Cold Genesys, Eisai, Engene, Ferring Pharmaceuticals, FerGene, Imagine Pharma, Janssen, MDxHealth, Medac, Merck, Pfizer, Photocure, ProTara Therapeutics, Roviant Sciences, Seattle Genetics, Sessen Bio, Theralase Technologies, TMC Innovation and US Biotest. A.M.K. has received grants and/or research support from Adolor Corporation, Bristol Myers Squibb, FKD Industries, Heat Biologics, Merck, Photocure, SWOG/NIH, Specialized Programs of Research Excellence (SPORE) and AIBCCR. A.M.K. also holds the patent for Cytokine Predictors of Response to Intravesical Therapy (CyPRIT) joint with UT MD Anderson Cancer Center. The other authors declare no competing interests.

Peer review information

Nature Reviews Urology thanks W. Krajewski, S. Vermeulen and the other, anonymous, reviewer(s) for their contribution to the peer review of this work.

Publisher's note

Springer Nature remains neutral with regard to jurisdictional claims in published maps and institutional affiliations.

(c) Springer Nature Limited 2021 